\title{
Comparison of stochastic and machine learning methods for multi-step ahead forecasting of hydrological processes
}

Georgia Papacharalampous ${ }^{1, *}$, Hristos Tyralis $^{2}$, and Demetris Koutsoyiannis ${ }^{3}$

1 Department of Water Resources and Environmental Engineering, School of Civil Engineering, National Technical University of Athens, Iroon Polytechniou 5, 15780 Zografou, Greece; papacharalampous.georgia@gmail.com; ORCID: 0000-0001-5446$954 \mathrm{X}$

2 Air Force Support Command, Hellenic Air Force, Elefsina Air Base, 19200 Elefsina, Greece; montchrister@gmail.com; ORCID: 0000-0002-8932-4997

3 Department of Water Resources and Environmental Engineering, School of Civil Engineering, National Technical University of Athens, Iroon Polytechniou 5, 15780 Zografou, Greece; dk@itia.ntua.gr; ORCID: 0000-0002-6226-0241

* Correspondence: papacharalampous.georgia@gmail.com, tel: +30 6947498589

This is a pre-print of an article published in Stochastic Environmental Research and Risk Assessment (2019). The final authenticated version is available online at: https://doi.org/10.1007/s00477-018-1638-6

Abstract: Research within the field of hydrology often focuses on the statistical problem of comparing stochastic to machine learning (ML) forecasting methods. The comparisons performed are based on case studies, while a study providing large-scale results on the subject is missing. Herein, we compare 11 stochastic and 9 ML methods regarding their multi-step ahead forecasting properties by conducting 12 extensive computational experiments based on simulations. Each of these experiments uses 2000 time series generated by linear stationary stochastic processes. We conduct each simulation experiment twice; the first time using time series of 100 values and the second time using time series of 300 values. Additionally, we conduct a real-world experiment using 405 mean annual river discharge time series of 100 values. We quantify the performance of the methods using 18 metrics. The results indicate that stochastic and ML methods perform equally well.

Key Words: no free lunch theorem; random forests; river discharge; stochastic hydrology; support vector machines; time series 


\section{Introduction}

\subsection{Background information}

The fundamental problem of statistically producing point forecasts of univariate time series by exploiting information from their past values only (hereafter, "forecasting", unless specified differently) is of traditional interest to hydrological scientists (Yevyevich 1987). Right after the introduction of the currently classical Autoregressive Integrated Moving Average (ARIMA) models by Box and Jenkins (1968), Carlson et al. (1970) used several stationary models of this specific family, i.e. Autoregressive Moving Average (ARMA) models, to forecast the evolution of four annual time series of streamflow processes. Today the available models for time series forecasting are numerous and can be classified according to De Gooijer and Hyndman (2006) into eight categories, i.e. (a) exponential smoothing, (b) ARIMA, (c) seasonal models, (d) state space and structural models and the Kalman filter, (e) nonlinear models, (f) long-range dependence models, e.g. the family of Autoregressive Fractionally Integrated Moving Average (ARFIMA) models, (g) Autoregressive Conditional Heteroscedastic/Generalized Autoregressive Conditional Heteroscedastic (ARCH/GARCH) models and (h) count data forecasting. The models from the categories (a)-(g) are of potential interest in hydrology, while they can be implemented for both one- and multi-step ahead forecasting.

The theoretical properties of the models of categories (a)-(d), (f), (g) (hereafter, referred to as "stochastic") have been more or less investigated, in contrast to those of the nonlinear models and in particular the Machine Learning (ML) algorithms, also referred to in the literature as black-box models. These two main categories of models are known to represent two different cultures in statistical modelling, the data modelling culture and the algorithmic modelling culture (Breiman 2001b). The former assumes that an analytically formulated stochastic model is behind the generation of the data, while the latter that behind this process is something complex and unknown, which does not have to be analytically formulated, as long as a purely algorithmic model can offer high forecast accuracy. In other words, profoundly understanding and properly modelling the (future) behaviour of a process are strongly connected within the data modelling culture, but completely irrelevant within the algorithmic modelling culture. The distinction between causal explanation, prediction and description is acknowledged and clarified in terms of modelling in Shmueli (2010). Still, one could question whether the (rather artificial) 
separation of models with respect to the "stochastic-ML dipole" actually corresponds to a striking difference in their forecasting performance.

What cannot be questioned, on the other hand, is the popularity that the various ML forecasting methods have gained in many scientific fields, including hydrology. Amongst the most popular ML algorithms are the Neural Networks (NN), Random Forests (RF) and Support Vector Machines (SVM). The SVM are presented in their current form by Cortes and Vapnik (1995) (see also Vapnik 1995, 1999), while RF by Breiman (2001a). For the implementation of the $\mathrm{NN}$ for time series forecasting the reader is referred to Zhang et al. (1998) and Zhang (2001). Regarding the use of SVM for this specific purpose, a review can be found in Sapankevych and Sankar (2009). The large number of the relevant applications of the NN and SVM algorithms in the field of hydrology is imprinted in Maier and Dandy (2000) and Raghavendra and Deka (2014) respectively, while Abrahart et al. (2012) collectively review the NN streamflow forecasting and rainfall-runoff applications (examples of the latter can be found, for instance, in De Vos (2013), although rainfallrunoff applications are a completely different problem since it is based on the use of exogenous variables, see also the discussion in Section 4). The RF algorithms, on the other hand, are barely used for the forecasting of hydrological processes.

In Table 1 we present some literature information on hydrometeorological time series forecasting to explore the related background and facilitate the following discussion. As it is apparent, hydrological research often focuses on ML or hybrid (e.g. a combination of ARMA and ML) forecasting methods and, in particular, on the comparison between stochastic (mainly ARMA and ARFIMA) and ML methods. However, the culture of assessing the performance of forecasting methods on large datasets is not customary in hydrology. Therefore, the assessment is performed within case studies. Concerning the testing procedure, while the available metrics for the assessment of the forecast quality are a lot, most of the studies use only a few (Krause et al. 2005), understating the importance of the testing process despite relevant suggestions (e.g. Humphrey et al. 2017). Similarly, the number of the implemented forecasting methods is usually small. 
Table 1. Case studies presenting forecasts of hydrometeorological processes.

\begin{tabular}{|c|c|c|c|c|c|c|c|c|c|c|c|c|c|}
\hline \multirow[t]{2}{*}{$\mathrm{s} / \mathrm{n}$} & \multirow[t]{2}{*}{ Study } & \multirow[t]{2}{*}{$\begin{array}{l}\text { Primary } \\
\text { focus }\end{array}$} & \multicolumn{4}{|c|}{$\begin{array}{l}\text { Hydrometeorological } \\
\text { process }\end{array}$} & \multicolumn{4}{|c|}{ Data level } & \multicolumn{3}{|c|}{ Horizon } \\
\hline & & & 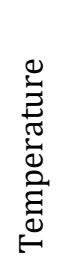 & 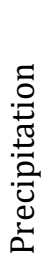 & 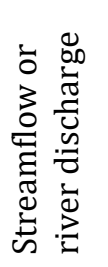 & $\frac{\dot{d}}{\stackrel{ \pm}{0}}$ & 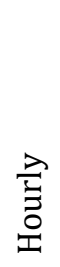 & $\frac{\lambda}{\vec{\pi}}$ & 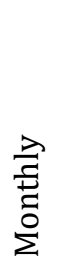 & 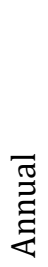 & 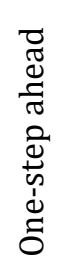 & 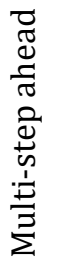 & 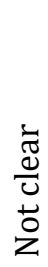 \\
\hline 1 & Atiya et al. (1999) & NN & $x$ & $x$ & $\checkmark$ & $x$ & $x$ & $x$ & $\checkmark$ & $x$ & $x$ & $\checkmark$ & $x$ \\
\hline 2 & $\begin{array}{l}\text { Lambrakis et al. } \\
(2000)\end{array}$ & methods & $x$ & $x$ & $\checkmark$ & $x$ & $\checkmark$ & $x$ & $x$ & $x$ & $\checkmark$ & $x$ & $x$ \\
\hline 3 & Kişi (2007) & & $x$ & $x$ & $\checkmark$ & $x$ & $x$ & $\checkmark$ & $x$ & $x$ & $\checkmark$ & $\checkmark$ & $x$ \\
\hline 4 & Cheng et al. (2008) & & $x$ & $x$ & $\checkmark$ & $\checkmark$ & $x$ & $x$ & $\checkmark$ & $\checkmark$ & $\checkmark$ & $\checkmark$ & $x$ \\
\hline 5 & Yaseen et al. (2016) & & $\times$ & $\times$ & $\checkmark$ & $\times$ & $x$ & $\checkmark$ & $\checkmark$ & $\times$ & $\checkmark$ & $x$ & $x$ \\
\hline 6 & $\begin{array}{l}\text { Sivapragasam et al. } \\
\text { (2001) }\end{array}$ & $\begin{array}{l}\text { SVM } \\
\text { methods }\end{array}$ & $x$ & $\sqrt{ }$ & $\checkmark$ & $x$ & $x$ & $\checkmark$ & $x$ & $x$ & $\checkmark$ & $x$ & $x$ \\
\hline 7 & Shi and Han (2007) & & $x$ & $x$ & $\checkmark$ & $\checkmark$ & $x$ & $x$ & $\checkmark$ & $\checkmark$ & $\checkmark$ & $\checkmark$ & $x$ \\
\hline 8 & Lu and Wang (2011) & & $\times$ & $\checkmark$ & $\times$ & $\times$ & $x$ & $\checkmark$ & $\times$ & $\times$ & $\checkmark$ & $x$ & $\times$ \\
\hline 9 & Hu et al. (2001) & Hybrid & $x$ & $\sqrt{ }$ & $x$ & $x$ & $x$ & $x$ & $x$ & $\checkmark$ & $\checkmark$ & $x$ & $x$ \\
\hline 10 & Kim and Valdés (2003) & methods & $x$ & $x$ & $x$ & $\checkmark$ & $x$ & $x$ & $\checkmark$ & $x$ & $\checkmark$ & $\checkmark$ & $x$ \\
\hline 11 & Pai and Hong (2007) & & $x$ & $\checkmark$ & $x$ & $x$ & $\checkmark$ & $x$ & $x$ & $x$ & $\checkmark$ & $x$ & $x$ \\
\hline 12 & Hong (2008) & & $x$ & $\checkmark$ & $x$ & $x$ & $\checkmark$ & $x$ & $x$ & $x$ & $\checkmark$ & $x$ & $x$ \\
\hline 13 & Kişi and Cimen (2011) & & $x$ & $\times$ & $\checkmark$ & $\times$ & $\times$ & $x$ & $\checkmark$ & $x$ & $\checkmark$ & $x$ & $\times$ \\
\hline 14 & $\begin{array}{l}\text { Liong and } \\
\text { Sivapragasam (2002) }\end{array}$ & $\begin{array}{l}\text { SVM vs NN } \\
\text { methods }\end{array}$ & $x$ & $x$ & $x$ & $\checkmark$ & $x$ & $\checkmark$ & $x$ & $x$ & $\checkmark$ & $\checkmark$ & $x$ \\
\hline 15 & Guo et al. (2011) & & $x$ & $x$ & $\checkmark$ & $x$ & $x$ & $x$ & $\checkmark$ & $x$ & $x$ & $x$ & $\checkmark$ \\
\hline 16 & Kişi and Cimen (2012) & & $x$ & $\checkmark$ & $x$ & $x$ & $x$ & $\checkmark$ & $x$ & $x$ & $\checkmark$ & $x$ & $x$ \\
\hline 17 & He et al. (2014) & & $x$ & $\times$ & $\checkmark$ & $\times$ & $x$ & $\checkmark$ & $\times$ & $\times$ & $\checkmark$ & $x$ & $x$ \\
\hline 18 & Jain et al. (1999) & Stochastic & $x$ & $x$ & $\checkmark$ & $x$ & $x$ & $x$ & $\checkmark$ & $x$ & $\checkmark$ & $x$ & $x$ \\
\hline 19 & Ballini et al. (2001) & vs ML & $x$ & $x$ & $\checkmark$ & $x$ & $x$ & $x$ & $\checkmark$ & $x$ & $\checkmark$ & $\checkmark$ & $x$ \\
\hline 20 & Kiși (2004) & methods & $x$ & $x$ & $\checkmark$ & $x$ & $x$ & $x$ & $\checkmark$ & $x$ & $\checkmark$ & $\checkmark$ & $x$ \\
\hline 21 & $\begin{array}{l}\text { Khan and Coulibaly } \\
\text { (2006) }\end{array}$ & & $x$ & $x$ & $x$ & $\checkmark$ & $x$ & $x$ & $\checkmark$ & $x$ & $\checkmark$ & $\checkmark$ & $x$ \\
\hline 22 & Lin et al. (2006) & & $x$ & $x$ & $\checkmark$ & $x$ & $x$ & $x$ & $\checkmark$ & $x$ & $x$ & $x$ & $\checkmark$ \\
\hline 23 & Mishra et al. (2007) & & $x$ & $x$ & $x$ & $\checkmark$ & $x$ & $x$ & $\checkmark$ & $x$ & $\checkmark$ & $\checkmark$ & $x$ \\
\hline 24 & Yu and Liong (2007) & & $x$ & $x$ & $\checkmark$ & $x$ & $x$ & $x$ & $x$ & $x$ & $x$ & $\checkmark$ & $x$ \\
\hline 25 & $\begin{array}{l}\text { Koutsoyiannis et al. } \\
\text { (2008) }\end{array}$ & & $x$ & $x$ & $\checkmark$ & $x$ & $x$ & $x$ & $\checkmark$ & $x$ & $\checkmark$ & $x$ & $x$ \\
\hline 26 & Wang et al. (2009) & & $x$ & $x$ & $\checkmark$ & $x$ & $x$ & $x$ & $\checkmark$ & $x$ & $x$ & $\checkmark$ & $x$ \\
\hline 27 & Abudu et al. (2010) & & $x$ & $x$ & $\checkmark$ & $x$ & $x$ & $x$ & $\checkmark$ & $x$ & $\checkmark$ & $x$ & $x$ \\
\hline 28 & Kişi et al. (2012) & & $x$ & $x$ & $x$ & $\checkmark$ & $x$ & $\checkmark$ & $x$ & $x$ & $\checkmark$ & $\checkmark$ & $x$ \\
\hline 29 & $\begin{array}{l}\text { Shabri and Suhartono } \\
\text { (2012) }\end{array}$ & & $x$ & $x$ & $\checkmark$ & $x$ & $x$ & $x$ & $\checkmark$ & $x$ & $\checkmark$ & $x$ & $x$ \\
\hline 30 & Valipour et al. (2013) & & $x$ & $x$ & $\checkmark$ & $x$ & $x$ & $x$ & $\checkmark$ & $x$ & $x$ & $x$ & $\checkmark$ \\
\hline 31 & $\begin{array}{l}\text { Patel and } \\
\text { Ramachandran (2014) }\end{array}$ & & $x$ & $x$ & $\checkmark$ & $x$ & $x$ & $x$ & $\checkmark$ & $x$ & $x$ & $\checkmark$ & $x$ \\
\hline 32 & $\begin{array}{l}\text { Papacharalampous et } \\
\text { al. (2017c) }\end{array}$ & & $\checkmark$ & $\checkmark$ & $x$ & $x$ & $x$ & $x$ & $\checkmark$ & $x$ & $\checkmark$ & $\checkmark$ & $x$ \\
\hline
\end{tabular}

Researchers have long been chasing the most accurate forecast for their data, a "universally best technique". On the other hand, there is an argument that it is the data and the application of interest that determine the proper methodology for each case, rather than vice versa (Hong and Fan 2016). Another argument is that perhaps research should 
invest more on probabilistic forecasting (e.g. using Bayesian statistics as in Tyralis and Koutsoyiannis 2014) and less on point forecasting (Krzysztofowicz 2001). In fact, the opinions on forecast evaluation are often diverging, as they tend to depend on the perspective from which the forecasts are examined. An interesting study on this subject can be found in Murphy (1993). The latter identifies three criteria for this specific evaluation, which are adopted as a foundation for further discussion in later studies, e.g. Ramos et al. (2010) and Weijs et al. (2010). These criteria are (1) the consistency during the forecasting process, (2) the quality or the correspondence between the forecasts and the target values and (3) the value or the profit that the forecast provide to the decision makers. Weijs et al. (2010) note that criterion (2) concerns more the pure science, while criterion (3) is closer related to the decisions made within the engineering applications (of science), rather than science itself. Thus, only a few studies are dedicated to criterion (3), such as Ramos et al. (2010) and Ramos et al. (2013), while the greatest part of the literature focuses on criterion (2). The latter likewise applies to the present study and to all of its references aiming to deal with the modelling issue (which model should I use?) within specific hydrological concepts.

Regarding the so far conducted comparisons between forecasting methods, their majority in all scientific fields is based on case studies. Nevertheless, in some few cases beyond the field of hydrology the number of the examined real-world time series is quite large. These time series are realizations of several phenomena, which however are fundamentally different from being hydrological, and their examination includes concepts that are rather inappropriate in hydrological terms (e.g. paying attention to small quantitative differences in the forecasting performance of the methods). Examples of such studies can be found in Makridakis et al. (1987), Makridakis and Hibon (2000) and Ahmed et al. (2010), which examine 1001,3003 and 1045 time series respectively. Within these studies a statistical analysis is performed and the results are presented correspondingly. Furthermore, the literature includes two studies (Zhang 2001; Thissen et al. 2003) in which the performance of the methods is assessed on simulated time series from linear stochastic processes. The scale of the simulation experiment is small in both cases. Thissen et al. (2003) examine one long time series from the ARMA family, while Zhang (2001) examine 8 stochastic processes from the ARMA family and 30 simulated time series for each stochastic process. The forecasting methods are ARMA models, NN and SVM in the former study and ARMA models and NN in the latter study, while Makridakis 
and Hibon (1987), Makridakis and Hibon (2000) and Ahmed et al. (2010) do not focus their comparisons on the stochastic-ML dipole.

Admittedly, the studies mentioned in the previous paragraph pursue generalized results to greater extent than most of the available studies. However, the gap still remains. What specifically needs to be addressed is whether the stochastic-ML dipole actually corresponds to a clear difference in the forecasting performance of the methods, especially in the light of published studies, which claim that they found a technique better than others. Given the fact that each forecasting case is indisputably unique, this task would necessarily require the examination of a sufficiently large and representative sample of forecasting cases within the same (properly designed) methodological framework. Some suggestions for the design of large-scale studies are available in Alpaydin (2010). Extensive simulations combined with statistical analysis and benchmarking can constitute, nevertheless, a highly effective approach to solving the problem under discussion. In more detail, for the generalized comparison of stochastic and ML forecasting methods, a sufficient number of different and representative of the underlying phenomena time series could be used for the estimation of the expected performance of several forecasting methods regarding several criteria of interest. The need of using simulated time series to assess the performance of forecasting methods is emphasized by forecasting experts (Bontempi 2013). The analytical approach in assessing the performance of ML algorithms is usually not possible; therefore, the only alternative approach is using simulations. Apparently, the larger the scale of the simulation experiments, the more general would be the results. Real-world experiments of large-scale could be used to complement the results of the simulation experiments in alignment with specific applications.

\subsection{The present study}

In the context described in the above section, we perform an extensive comparison between several stochastic and ML methods for the forecasting of hydrological processes by conducting large-scale computational experiments based on simulations. The comparison refers to the multi-step ahead forecasting properties of the methods, although one-step ahead forecasting is also of practical and scientific interest. The simulated time series are 48000 in total, while they are generated by linear stationary stochastic processes. The latter are commonly used for modelling hydrological processes. 
In fact, stationary models, in contrast to the non-stationary, are established as the appropriate modelling choice when dealing with natural processes, unless tangible and quantitative information that can fully support a deterministic description (not based on data but on physical laws) of change in time are available (Koutsoyiannis 2011; Koutsoyiannis and Montanari 2015). Additionally to the simulation experiments, we examine 405 real-world time series. Our aim is to fill the gap detected in the literature by providing large-scale results and useful insights on the comparison of stochastic and ML forecasting methods for the case of hydrological time series forecasting, with an emphasis on river discharge processes. A strength (and limitation) of the present study (implied by its aim) is the adopted approach to the problem, i.e. the algorithmic one.

The present study was first presented by Papacharalampous et al. (2017a), while a preliminary research on the subject was conducted for the Postgraduate Thesis of the first author (Papacharalampous 2016). Subsequently, we provide some basic information about the large-scale companion studies of this paper. Papacharalampous et al. (2017b) examine the problem of error evolution in hydrological multi-step ahead forecasting, while Tyralis and Papacharalampous (2017) improve the performance of RF in one-step ahead forecasting of geophysical processes. Papacharalampous et al. (2018b) also focuses on the problem of one-step ahead forecasting with the aim to provide large-scale results on the latter in geoscience. These three studies examine simulated, as well as real-world datasets. In detail, they examine 12000 simulated and 92 monthly streamflow time series, 16000 simulated and 135 annual temperature time series, and 24000 simulated, 185 annual temperature and 112 annual precipitation time series respectively. Finally, Papacharalampous et al. (2018c) produce multi-step ahead forecasts for 985 monthly temperature and 1552 monthly precipitation time series aiming at the investigation of the predictability of these processes. All the time series examined by the present study and its companions are short, as it is expected for the hydrometeorological time series.

A subsequent to Papacharalampous (2016) and Papacharalampous et al. (2017a) study by Makridakis et al. (2018) focuses on a similar investigation to the present paper, although in a different field and under a different experimental setting. In particular Makridakis et al. (2018) conclude that at the moment the statistical methods are better compared to the machine learning methods, while as shown here the performance of all methods is similar (see also the discussion of Section 4 for the Makridakis et al. (2018) paper and the implications of the results of the present study in geosciences). 


\section{Methodology}

In Section 2 we present the basic methodological elements of this study, while the reader is referred to the supplementary material for a brief theoretical background, as also to the scientific literature for a more complete coverage of the relevant theory.

\subsection{Simulated processes}

We simulate time series according to several stochastic models from the frequently used families of ARMA and ARFIMA. This modelling approach is considered appropriate for the achievement of our aim and has been widely applied in hydrology (e.g. Montanari et al. (1997, 2000), Ballini et al. (2001), Wang et al. (2009), Valipour et al. (2013)). The simulated stochastic processes are presented in Table 2, while for the related definitions the reader is referred to the report entitled "Definition of the stochastic processes" of the supplementary material. These 12 stochastic models correspond to different types of autocorrelation. We use the arima.sim built in R algorithm ( $R$ Core Team 2017) to simulate the $\operatorname{ARMA}(p, q)$ processes and the fracdiff.sim algorithm of the fracdiff $\mathrm{R}$ package (Fraley et al. 2012) to simulate the $\operatorname{ARFIMA}(p, d, q)$ processes.

Table 2. Simulated stochastic processes of the present study. Their definitions are given in the supplementary material.

\begin{tabular}{lll}
\hline $\mathrm{s} / \mathrm{n}$ & Stochastic model & Parameters of the stochastic model \\
\hline 1 & AR(1) & $\varphi_{1}=0.7$ \\
2 & AR(1) & $\varphi_{1}=-0.7$ \\
3 & AR(2) & $\varphi_{1}=0.7, \varphi_{2}=0.2$ \\
4 & MA(1) & $\theta_{1}=0.7$ \\
5 & MA(1) & $\theta_{1}=-0.7$ \\
6 & ARMA(1,1) & $\varphi_{1}=0.7, \theta_{1}=0.7$ \\
7 & ARMA(1,1) & $\varphi_{1}=-0.7, \theta_{1}=-0.7$ \\
8 & ARFIMA(0,0.45,0) & \\
9 & ARFIMA(1,0.45,0) & $\varphi_{1}=0.7$ \\
10 & ARFIMA(0,0.45,1) & $\theta_{1}=-0.7$ \\
11 & ARFIMA(1,0.45,1) & $\varphi_{1}=0.7, \theta_{1}=-0.7$ \\
12 & ARFIMA(2,0.45,2) & $\varphi_{1}=0.7, \varphi_{2}=0.2, \theta_{1}=-0.7, \theta_{2}=-0.2$ \\
\hline
\end{tabular}




\subsection{Real-world time series}

We examine 405 mean annual discharge time series of 100 values, sourced from GRDC (2017). For the exploration of these time series we calculate the sample Autocorrelation Function (ACF) and the sample Partial Autocorrelation Function (PACF). The side-by-side boxplots of the ACF and PACF estimates are presented in Figure 1. The Hurst-Kolmogorov behaviour (HK behaviour) is a common property of geophysical properties (e.g. Tyralis and Koutsoyiannis 2011). To describe the HK behaviour of discharge we estimate the Hurst parameter $H$ of all time series using the mleHK algorithm of the HKprocess $\mathrm{R}$ package (Tyralis 2016), which implements the maximum likelihood method (Tyralis and Koutsoyiannis 2011). The parameter $H$ takes values in the interval $(0,1)$. The larger it is the larger the magnitude of the HK behaviour, which can be modelled by an $\operatorname{ARFIMA}(0, d, 0)$ model. A histogram of the $H$ estimates is presented in Figure 1 . By the examination of the latter we observe that the magnitude of the long-range dependence in the real-world time series is mostly significant. 
(a)

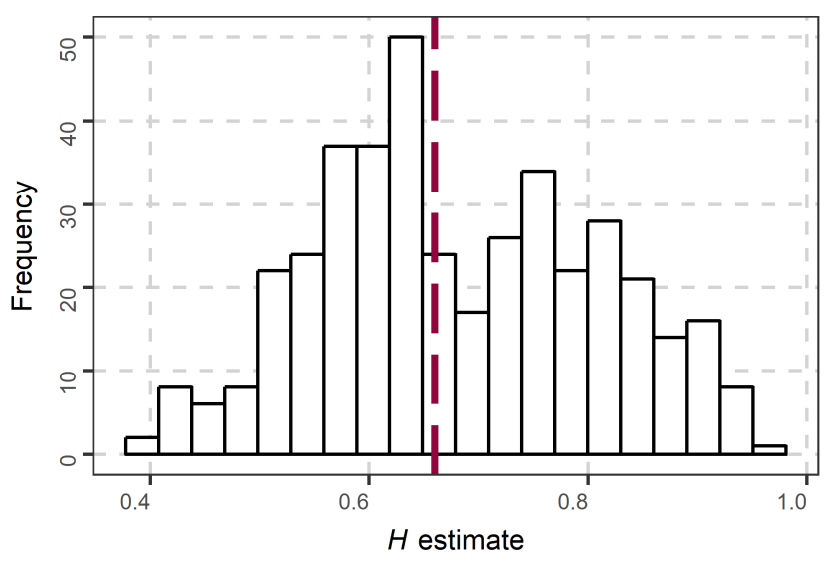

(b)

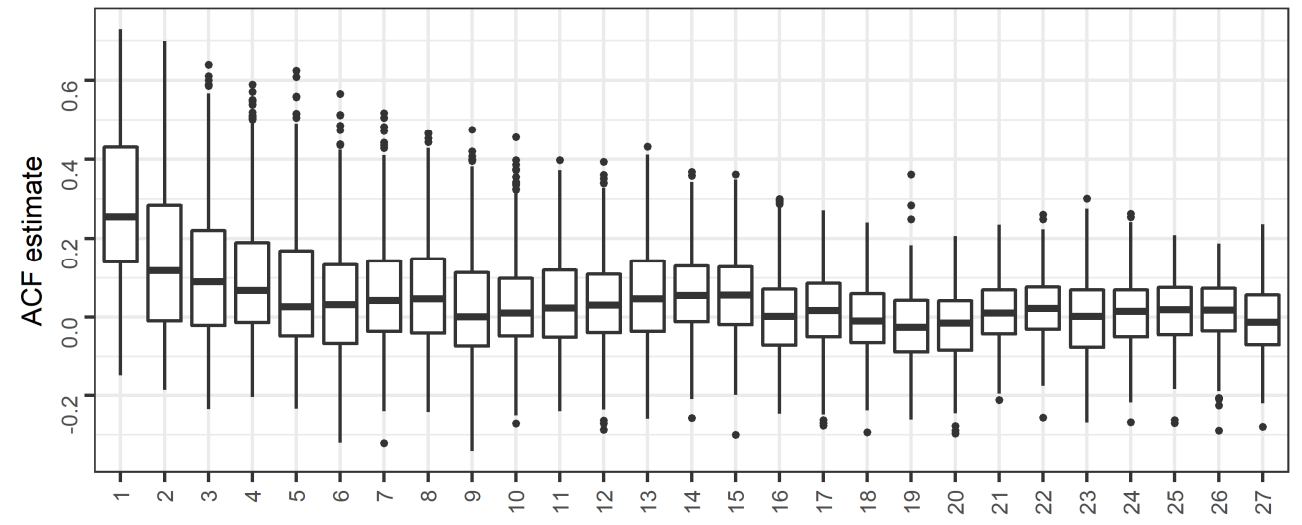

(c)

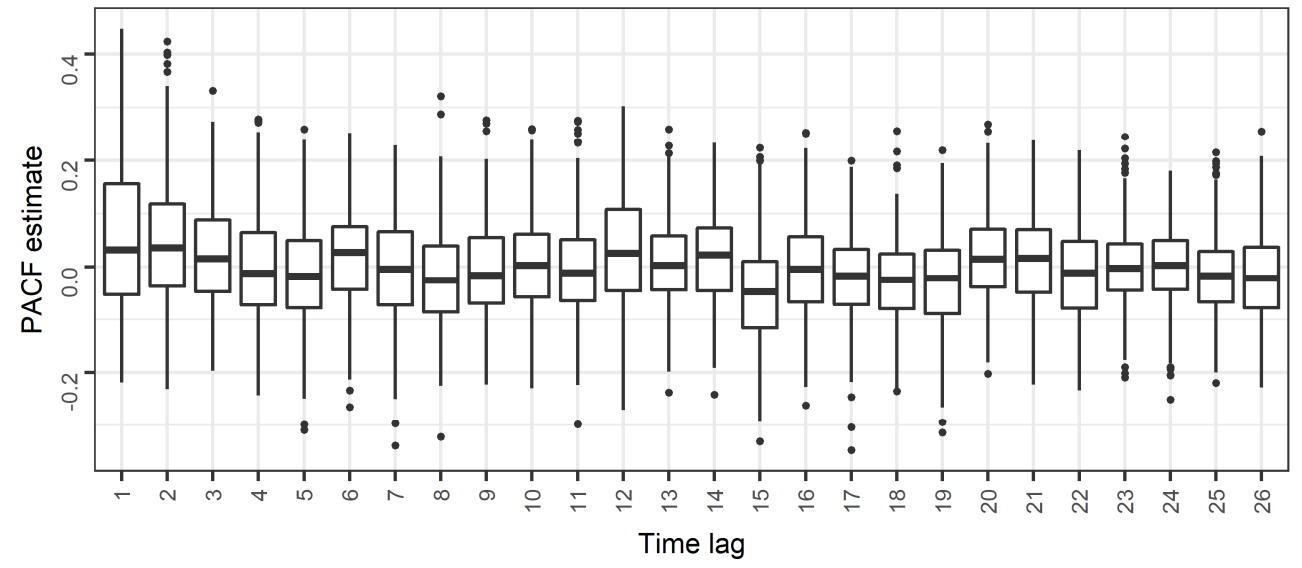

Figure 1. (a) $H$, (b) ACF, (c) PACF estimates of the real-world time series. Data source: GRDC (2017). The red dashed line in the upper graph denotes the median of the $H$ estimates.

\subsection{Forecasting methods}

We compare 11 stochastic to 9 ML forecasting methods. The stochastic methods are classified into five main categories as presented in Table 3. Similarly, the ML methods are classified into three main categories as presented in a compact form in Table 4 and Table 5 , while further details are omitted for reasons of brevity. For the implementation of the forecasting methods the reader is referred to the supplementary material. 
Table 3. Stochastic forecasting methods. The forecasting methods are available in code form in the supplementary material.

\begin{tabular}{lll}
\hline s/n & Abbreviated name & Category \\
\hline 1 & Naïve & Simple \\
2 & RW & \\
\hline 3 & ARIMA_f & ARIMA \\
4 & ARIMA_s & \\
5 & auto_ARIMA_f & \\
6 & auto_ARIMA_s & \\
\hline 7 & auto_ARFIMA & ARFIMA \\
\hline 8 & BATS & State Space \\
9 & ETS_S & \\
\hline 10 & SES & Exponential Smoothing \\
11 & Theta & \\
\hline
\end{tabular}

Table 4. ML forecasting methods. The time lag selection procedures adopted are defined in Table 5. The forecasting methods are available in code form in the supplementary material.

\begin{tabular}{|c|c|c|c|c|c|}
\hline $\mathrm{s} / \mathrm{n}$ & $\begin{array}{l}\text { Abbreviated } \\
\text { name }\end{array}$ & Category & $\begin{array}{l}\text { Model structure } \\
\text { information }\end{array}$ & $\begin{array}{l}\text { Hyperparameter } \\
\text { optimized } \\
\text { (grid values) }\end{array}$ & $\begin{array}{l}\text { Time lag } \\
\text { selection } \\
\text { procedure }\end{array}$ \\
\hline 1 & NN_1 & \multirow[t]{3}{*}{ NN } & Single hidden layer & Number of hidden & 1 \\
\hline 2 & NN_2 & & Multilayer & nodes $(0,1, \ldots, 15)$ & 2 \\
\hline 3 & NN_3 & & Perceptron (MLP) & & 3 \\
\hline 4 & RF_1 & \multirow[t]{3}{*}{$\mathrm{RF}$} & \multirow{3}{*}{$\begin{array}{l}\text { Breiman' s random } \\
\text { forests algorithm } \\
\text { with } 500 \text { grown } \\
\text { trees }\end{array}$} & \multirow{3}{*}{$\begin{array}{l}\text { Number of variables } \\
\text { randomly sampled } \\
\text { as candidates at each } \\
\text { split }(1, \ldots, 5)\end{array}$} & 1 \\
\hline 5 & RF_2 & & & & 2 \\
\hline 6 & RF_3 & & & & 3 \\
\hline 7 & SVM_1 & \multirow[t]{3}{*}{ SVM } & Radial Basis kernel & Sigma inverse & 1 \\
\hline 8 & SVM_2 & & "Gaussian" function, & kernel width & 2 \\
\hline 9 & SVM_3 & & $C=1$, epsilon $=0.1$ & $\left(2^{n}, n=-8,-7, \ldots, 6\right)$ & 3 \\
\hline
\end{tabular}

Table 5. Time lag selection procedures adopted for the ML methods. The forecasting methods are available in code form in the supplementary material.

\begin{tabular}{ll}
\hline $\mathrm{s} / \mathrm{n}$ & Time lags \\
\hline 1 & $\begin{array}{l}\text { The corresponding to an estimated value for the ACF using the acf R algorithm (built } \\
\text { in R algorithm), i.e. the time lags } 1, \ldots, 19 \text { for a time series of } 90 \text { values and the time }\end{array}$ \\
& $\begin{array}{l}\text { lags } 1, \ldots, 24 \text { for a time series of } 290 \text { values } \\
2\end{array}$ \\
& $\begin{array}{l}\text { The corresponding to a statistical important estimated value for the ACF using the acf } \\
\text { R algorithm (built in R algorithm). If there is no statistical important estimated value }\end{array}$ \\
& $\begin{array}{l}\text { for the ACF, the corresponding to the largest estimated value } \\
\text { According to the nnetar R function (package forecast), i.e. the time lags } 1, \ldots, n, \text { where } \\
n \text { is the number of AR parameters that are fitted to the time series data using the ar R } \\
\text { algorithm (built in R algorithm) }\end{array}$
\end{tabular}

We use two simple forecasting methods in the comparisons. The Naïve forecasting method, one of the most commonly used benchmarks (Hyndman and Athanasopoulos 2013; Pappenberger et al. 2015), simply sets all forecasts equal to the last value. The RW forecasting method, a variation of the Naïve forecasting method, is equivalent to drawing a line between the first and the last value and extrapolating it into the future (Hyndman 
and Athanasopoulos 2013). The stochastic methods also include the ARIMA and ARFIMA methods. These five methods apply the maximum likelihood method to estimate the values of the parameters of the AR and MA parts of the models. For the ARIMA_f and ARIMA_s forecasting methods the numbers of the AR $(p)$ and MA $(q)$ parameters are set to be the same to those used in the simulated processes, while the number of differencing (d) is set to be zero. The auto_ARIMA_f and auto_ARIMA_s methods estimate the values of $p, d, q$ of the ARIMA model using the Akaike Information Criterion with a correction for finite sample sizes (AICc), as described in Hyndman and Athanasopoulos (2013). The same applies to the auto_ARFIMA method for the estimation of the values of $p, d, q$ of the ARFIMA models. We note that ARIMA_s and auto_ARIMA_s are simulation models.

The BATS and ETS_s forecasting methods use the point forecasts from an exponential smoothing state space model with several key features, i.e. capability of performing BoxCox transformation and/or including ARMA errors correction, Trend and Seasonal components (BATS), also allowing an optimal model selection using the Akaike Information Criterion (AIC), and an exponential smoothing state space simulation model with automatic selection of the Error, Trend and Seasonal components (ETS) respectively. We additionally include the SES (Simple Exponential Smoothing) and Theta forecasting methods in the comparisons. The latter method was presented by Assimakopoulos and Nikolopoulos (2000) and performed well in the M3-Competition (Makridakis and Hibon 2000). The reader is referred to Hyndman et al. (2008) and Hyndman and Athanasopoulos (2013) for the theoretical background of the exponential smoothing and space state models.

Regarding the NN, the RF and the SVM forecasting methods, there are some additional concerns to the selection of the algorithms, originating from the nature of the ML methods. The choices to be considered for the selection of the time lags used to build the regression matrix (input data matrix), as well as the choices for the values of the hyperparameters of the models (e.g. the hidden nodes in a NN model), are many. Usually, hyperparameters are not automatically decided by the ML algorithm during the fitting process. A fact is that the ML models are by design rather more flexible than needed in most cases and, thus, hyperparameter optimization is often used to detect and prevent overfitting as much as possible. In Tables 4 and 5 we summarize the basic information about the model structures, the hyperparameter optimization and the time lag selection procedures adopted. 
We apply the stochastic methods using mainly the R package forecast (Hyndman and Khandakar 2008, Hyndman et al. 2017) and the ML methods using the R package rminer (Cortez 2010, 2016) and the nnetar algorithm from the $\mathrm{R}$ package forecast (the latter is the NN_3 forecasting method), as also several built in R algorithms. The R package rminer uses the nnet algorithm of the nnet R package (Venables and Ripley 2002), the randomForest algorithm of the randomForest R package (Liaw and Wiener 2002) and the ksvm algorithm of the kernlab R package (Karatzoglou et al. 2004) for the application of the NN, the RF and the SVM methods respectively.

\subsection{Metrics}

The metrics used for the comparative assessment of the forecasting methods are classified into five main categories according to the criteria of Table 6. They provide assessment regarding two types of accuracy, the capture of the variance and the correlation. By Type 1 accuracy we mean the closeness of the forecasted time series to the actual, while by Type 2 accuracy we mean the closeness of the mean of the forecasted values of each time series to the mean of the actual ones. The definitions of the metrics are listed in the report entitled "Definition of the metrics" of the supplementary material, while the reader is also referred to Nash and Sutcliffe (1970), Kitanidis and Bras (1980), Yapo et al. (1996), Krause et al. (2005), Criss and Winston (2008), Gupta et al. (2009), Zambrano-Bigiarini (2014) for further information.

Table 6. Metrics used in the present study. Their definitions are given in the supplementary material.

\begin{tabular}{|c|c|c|c|c|c|c|}
\hline $\mathrm{s} / \mathrm{n}$ & $\begin{array}{l}\text { Abbreviated } \\
\text { Name }\end{array}$ & Full name & Criterion & Values & $\begin{array}{l}\text { Optimum } \\
\text { Value }\end{array}$ & $\begin{array}{l}\text { Condition } \\
\text { (the desired) }\end{array}$ \\
\hline 2 & MAPE & Mean Absolute Percentage Error & & {$[0,+\infty)$} & 0 & smaller MAPE \\
\hline 4 & NSE & Nash-Sutcliffe Efficiency & & $(-\infty, 1]$ & 1 & larger NSE \\
\hline 5 & mNSE & Modified Nash-Sutcliffe Efficiency & & $(-\infty, 1]$ & 1 & larger mNSE \\
\hline 6 & rNSE & Relative Nash-Sutcliffe Efficiency & & $(-\infty, 1]$ & 1 & larger rNSE \\
\hline 8 & ME & Mean Error & Type 2 accuracy & $(-\infty,+\infty)$ & 0 & smaller |ME| \\
\hline 9 & MPE & Mean Percentage Error & & $(-\infty,+\infty)$ & 0 & smaller |MPE| \\
\hline 10 & PBIAS & Percent Bias & & $(-\infty,+\infty)$ & 0 & smaller |PBIAS| \\
\hline 11 & VE & Volumetric Efficiency & & $(-\infty,+\infty)$ & 1 & smaller |VE - 1| \\
\hline 12 & rSD & Ratio of Standard Deviations & $\begin{array}{l}\text { Capture of the } \\
\text { variance }\end{array}$ & {$[0,+\infty)$} & 1 & $\begin{array}{l}\text { larger } \min \{r S D, \\
1 / \mathrm{rSD}\}\end{array}$ \\
\hline 16 & md & Modified Index of Agreement & & {$[0,1]$} & 1 & larger md \\
\hline 17 & $\mathrm{rd}$ & Relative Index of Agreement & & $(-\infty, 1]$ & 1 & larger rd \\
\hline 18 & KGE & Kling-Gupta Efficiency & $\begin{array}{l}\text { Type } 2 \text { accuracy, } \\
\text { capture of the } \\
\text { variance, correlation }\end{array}$ & $(-\infty, 1]$ & 1 & larger KGE \\
\hline
\end{tabular}




\subsection{Methodology outline}

For the comparison of the forecasting methods (see Section 2.3) we conduct 12 large-scale computational experiments based on simulations. Within each of the latter we simulate 2000 time series according to a stochastic process (see Section 2.1). We conduct each simulation experiment twice; the first time using time series of 100 values and the second time using time series of 300 values. The simulation experiments are named as presented in Table 7. Additionally, we conduct a real-world experiment using the time series presented in Section 2.2. We apply the forecasting methods to the simulated and the realworld time series according to Table 8 . The total number of forecasts is 858480 , among which 6480 are produced within the real-world experiment.

Table 7. Simulation experiments of the present study. The simulated processes are presented in Table 2.

\begin{tabular}{llll}
\hline s/n & Code & Simulated process & Length of the time series \\
\hline 1 & SE_1a & 1 & 100 values \\
2 & SE_2a & 2 & \\
3 & SE_3a & 3 & \\
4 & SE_4a & 4 & \\
5 & SE_5a & 5 & \\
6 & SE_6a & 6 & \\
7 & SE_7a & 7 & \\
8 & SE_8a & 8 & \\
9 & SE_9a & 9 & \\
10 & SE_10a & 10 & \\
11 & SE_11a & 11 & \\
12 & SE_12a & 12 & \\
\hline 13 & SE_1b & 1 & \\
14 & SE_2b & 2 & \\
15 & SE_3b & 3 & \\
16 & SE_4b & 4 & \\
17 & SE_5b & 5 & \\
18 & SE_6b & 6 & \\
19 & SE_7b & 7 & \\
20 & SE_8b & 8 & \\
21 & SE_9b & 9 & \\
22 & SE_10b & 10 & \\
23 & SE_11b & 11 & \\
24 & SE_12b & 12 & \\
\hline
\end{tabular}


Table 8. Use of the forecasting methods on the time series.

\begin{tabular}{llll}
\hline Forecasting method & ARMA simulated processes & ARFIMA simulated process & Real-world time series \\
\hline Naïve & $\checkmark$ & $\checkmark$ & $\checkmark$ \\
RW & $\checkmark$ & $\checkmark$ & $\checkmark$ \\
ARIMA_f & $\checkmark$ & $\times$ & $\times$ \\
ARIMA_s & $\checkmark$ & $\times$ & $\times$ \\
auto_ARIMA_f & $\checkmark$ & $\times$ & $\times$ \\
auto_ARIMA_s & $\checkmark$ & $\times$ & $\times$ \\
auto_ARFIMA & $\times$ & $\checkmark$ & $\checkmark$ \\
BATS & $\checkmark$ & $\checkmark$ & $\checkmark$ \\
ETS_s & $\checkmark$ & $\checkmark$ & $\checkmark$ \\
SES & $\checkmark$ & $\checkmark$ & $\checkmark$ \\
Theta & $\checkmark$ & $\checkmark$ & $\checkmark$ \\
\hline NN_1 & $\checkmark$ & $\checkmark$ & $\checkmark$ \\
NN_2 & $\checkmark$ & $\checkmark$ & $\checkmark$ \\
NN_3 & $\checkmark$ & $\checkmark$ & $\checkmark$ \\
RF_1 & $\checkmark$ & $\checkmark$ & $\checkmark$ \\
RF_2 & $\checkmark$ & $\checkmark$ & $\checkmark$ \\
RF_3 & $\checkmark$ & $\checkmark$ & $\checkmark$ \\
SVM_1 & $\checkmark$ & $\checkmark$ & $\checkmark$ \\
SVM_2 & $\checkmark$ & $\checkmark$ & $\checkmark$ \\
SVM_3 & $\checkmark$ & $\checkmark$ & $\checkmark$ \\
\hline
\end{tabular}

For the application of the stochastic methods we divide each time series into two segments, i.e. the fitting segment and the test segment, which contain $n_{1}$ and $n_{2}$ values respectively, as indicated in Figure 2(a). We fit the stochastic models to the former and make predictions corresponding to the latter using the recursive multi-step ahead forecasting method. For the total of the conducted experiments $n_{2}$ equals 10 . For the application of the ML forecasting methods, we additionally divide the segment of $n_{1}$ values into two segments, i.e. the fitting segment (first [2n1/3] values of the time series) and the validation segment, as also indicated in Figure 2(b). 
(a)

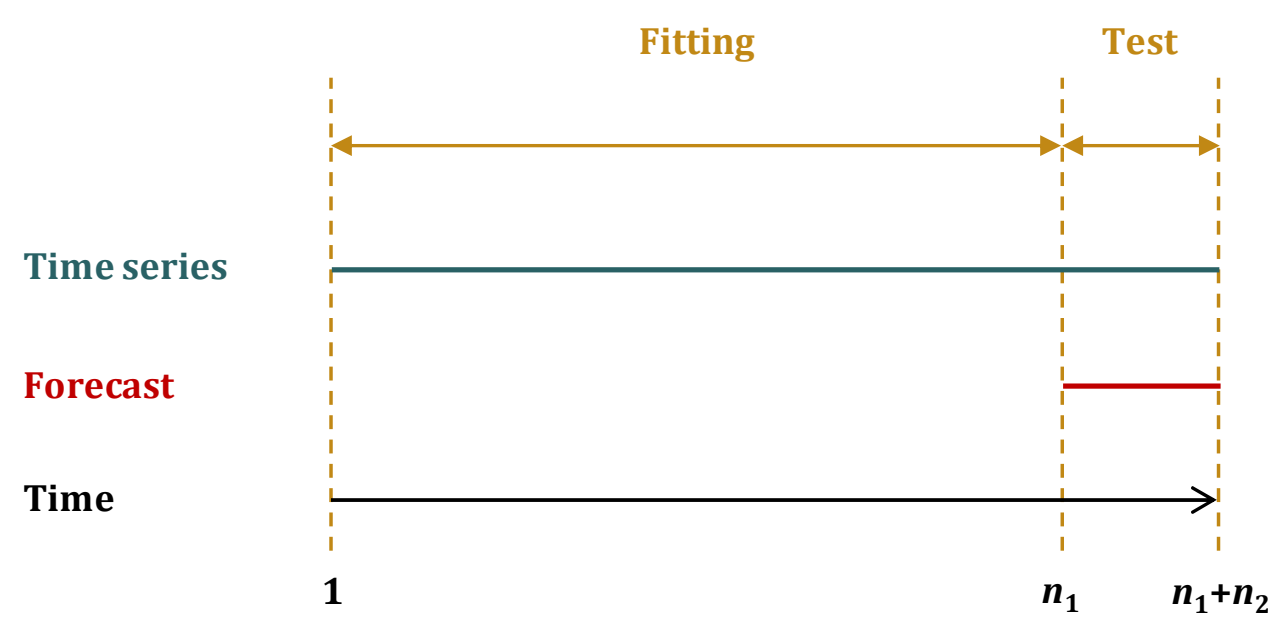

(b)

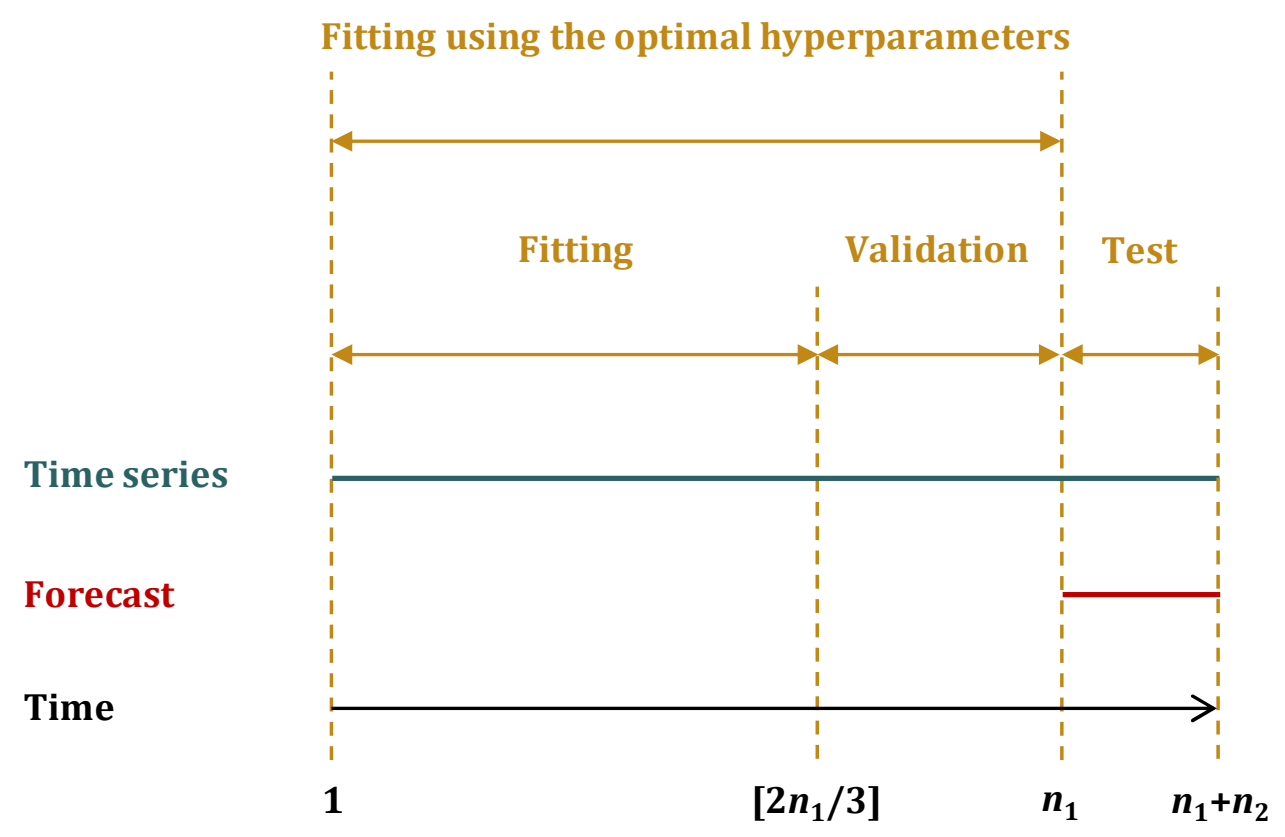

Figure 2. Division of a time series into (a) two segments for the application of the stochastic methods and (b) three segments for the application of the ML methods. We note that for the latter category the validation set serves the hyperparameter optimization procedure, while both the fitting and validation segments are used to re-fit the optimum models (i.e. the models with the optimal hyperparameters) before forecasting the values of the test segment.

The validation segment serves the hyperparameter optimization procedure, as explained subsequently. We use the fitting segment to fit several ML models that differ only as it comes to the values of a specific hyperparameter. We use each of those models to make predictions corresponding to the validation segment and measure the RMSE of those predictions. Finally, we decide on the value of the hyperparameter, i.e. the corresponding to the model with the smallest RMSE on the validation segment (optimum 
model). We fit a model with the selected hyperparameter value to data of both the fitting and validation segments and make predictions corresponding to the test segment.

Finally, we compute the values of the metrics presented in Section 2.4 for each forecasting test. The computation takes place on the test segment, which functions as a reference for the comparative assessment of the forecasting methods' performance. We use the metric values for the comparative assessment of the forecasting methods, mainly their medians and iqr values computed for each method per experiment. We compare the medians within each experiment, as described in Table 6, while the smallest the iqr the better the forecasts. In particular, for the real-world experiment we rank the forecasting methods for each individual test and further compute an average-case ranking for each of the metrics. We emphasize in the 18 average-case rankings and not directly in the mean or median values of the metrics (as in Tyralis and Papacharalampous 2017), because the latter might be more affected by the results of specific time series.

Although our computational experiments are designed to produce new knowledge in the field of hydrological time series forecasting, there are several outcomes rather well known at the forefront of our methodological framework. In more detail, the ARIMA_f and also the auto_ARIMA_f forecasting methods are expected to have the best performance regarding the Type 1 accuracy, mainly in terms of RMSE, on the time series resulting from the simulation of ARMA processes because of their theoretical background (for details see Wei 2006, pp. 88-93). Likewise, this applies to the performance of ARIMA_s and auto_ARIMA_s regarding the capture of the variance exhibited by the time series within the same simulation experiments. Furthermore, the ARIMA_f and ARIMA_s forecasting methods share an additional advantage, since they use by design the $\mathrm{p}$, $\mathrm{d}$, q numbers used in the simulation process. Similarly to the ARIMA_f and auto_ARIMA_f forecasting methods, auto_ARFIMA is expected to be the best in terms of RMSE on the time series resulting from the simulation of ARFIMA processes. The five forecasting methods mentioned in the present paragraph, together with the two simple methods, play the role of benchmarks within our methodological approach, while simulation experiments are, in general, the only available way to incorporate benchmarking into a methodological framework. 


\section{Results}

\subsection{Simulation experiments}

Section 3.1 aims at providing a synopsis of the results of the simulation experiments. To support our key findings, here we present a small representative sample of the entire information. For the about 13000 figures, conducted in the context of an exploratory visualization, as well as for the numerical summaries of the results in table form, the reader is referred to the fully reproducible reports, which are available together with their codes in the supplementary material. In the latter we also enclose the report entitled "Selected figures for the qualitative comparison of the forecasting methods", which includes Figures S.1-S.24. These figures can support the main conclusions of this paper in a satisfactory manner.

In Figures 3-5 we present the side-by-side boxplots of the metric values computed within the SE_1a simulation experiment. These figures can provide a rough outline of the forecasting methods and the utility of the metrics within this study. By their examination, we observe that the ARIMA_f and auto_ARIMA_f benchmarks are the best performing with respect to Type 1 accuracy, as assumed in Section 2.5, while BATS exhibits a very close to these methods performance, perhaps because it uses information from an ARMA model. We also note that the total of the ML methods except for NN_1 are competitive with BATS and with each other, while they are also better than the stochastic SES and Theta. The latter forecasting methods share a quite similar performance, a fact also applying to Naïve and RW. These simple benchmarks are better than NN_1 and the simulation models (ARIMA_s, auto_ARIMA_s, ETS_s), amongst which ETS_s produces forecasts with the most varying metric values and the worst median. Regarding the Type 2 accuracy, all the methods seem to have rather equally good average-case performance, since the differences in the latter are small and not perceivable from these figures. However, the metric values computed for ETS_s are the most scattered with respect to each other, while the opposite applies to the metric values computed for ARIMA_f, auto_ARIMA_f, BATS and all the ML methods apart from NN_1. The metric values computed for the remaining forecasting methods are scattered with respect to each other to an extent in between.

In terms of $\mathrm{rSD}$, the image is rather reversed compared to the one produced by the Type 1 accuracy metrics. Naïve, RW, SES and Theta are clearly the worst, while the ML methods are more segregated. The average-case performance of NN_1, ARIMA_f, 
auto_ARIMA_f and ETS_s is good. Nevertheless, the rSD values for these four forecasting methods can vary significantly from the one forecasting attempt to the other, more than the rSD values computed for the remaining forecasting methods, a fact also applying to the rest of the metrics. Regarding the average-case performance with respect to correlation, ARIMA_f, auto_ARIMA_f and BATS are the best, followed by NN_3. With respect to Type 1 accuracy and capture of the variance, ARIMA_f, auto_ARIMA_f, BATS and all the ML methods apart from NN_1 are clearly better than the simple benchmarks and competitive with each other. SES and Theta, on the other hand, exhibit a very close performance to the one of Naïve and RW. Finally, in terms of KGE, the best performing methods are the same three stochastic and eight ML ones. NN_1, ARIMA_f and auto_ARIMA_f are better than Theta, which is competitive with RW. Overall, we observe that for the SE_1a simulation experiment the metrics (even the corresponding to the same criterion) provide different aspects of the same information to an extent larger or smaller, while these 18 different aspects may also be conflicting to each other.

Subsequently, we state the main observations obtained from the total of the simulation experiments. To base these observations, in Figure 6 we present the heatmaps of the average-case performance of the forecasting methods within the SE_1a, SE_1b, SE_2a and SE_2b simulation experiments, while in Figures 7-9 we present the heatmaps formed using the medians of the total of the RMSE, rSD and d metric values respectively. In these figures the scaling is performed in the row direction and the darker the colour the better the forecasts. A clustering analysis on the forecasting methods based on their performance is also applied. Some observations obtained from SE_1a apply to the rest of the simulation experiments as well. These are the following (see, for example, Figures 69): (a) forecasting methods from both the stochastic and ML categories are amongst the best performing and the worst performing ones, (b) the metrics can provide significantly different, even conflicting, image regarding the performance of the forecasting methods, (c) the ARIMA_f, auto_ARIMA_f and auto_ARFIMA benchmarks are the best performing in terms of Type 1 accuracy, while ETS_s, and the ARIMA_f and auto_ARIMA_f benchmarks exhibit a good average-case performance in terms of rSD, (d) the image produced by rSD is reversed with respect to the one produced by the Type 1 accuracy metrics, i.e. the well performing with respect to the latter criterion are bad performing with respect to the capture of the variance of the time series, (e) BATS is very close to the ARIMA_f, auto_ARIMA_f and auto_ARFIMA benchmarks, and (f) Naïve and RW, as well as SES and 
Theta, exhibit similar performance with each other. Nevertheless, the Pr, r2 and KGE values computed for Naïve and SES are infinite. Finally, by the examination of the side-byside boxplots produced for each and every of the simulation experiments we note that $(\mathrm{g})$ the ARIMA_s, auto_ARIMA_s, ETS_S and NN_1 forecasting methods seem to share a form of instability, i.e. their metric values vary more with each other than the metric values of other forecasting methods. The latter concerns the results obtained from all the metrics except for Pr and r2. 

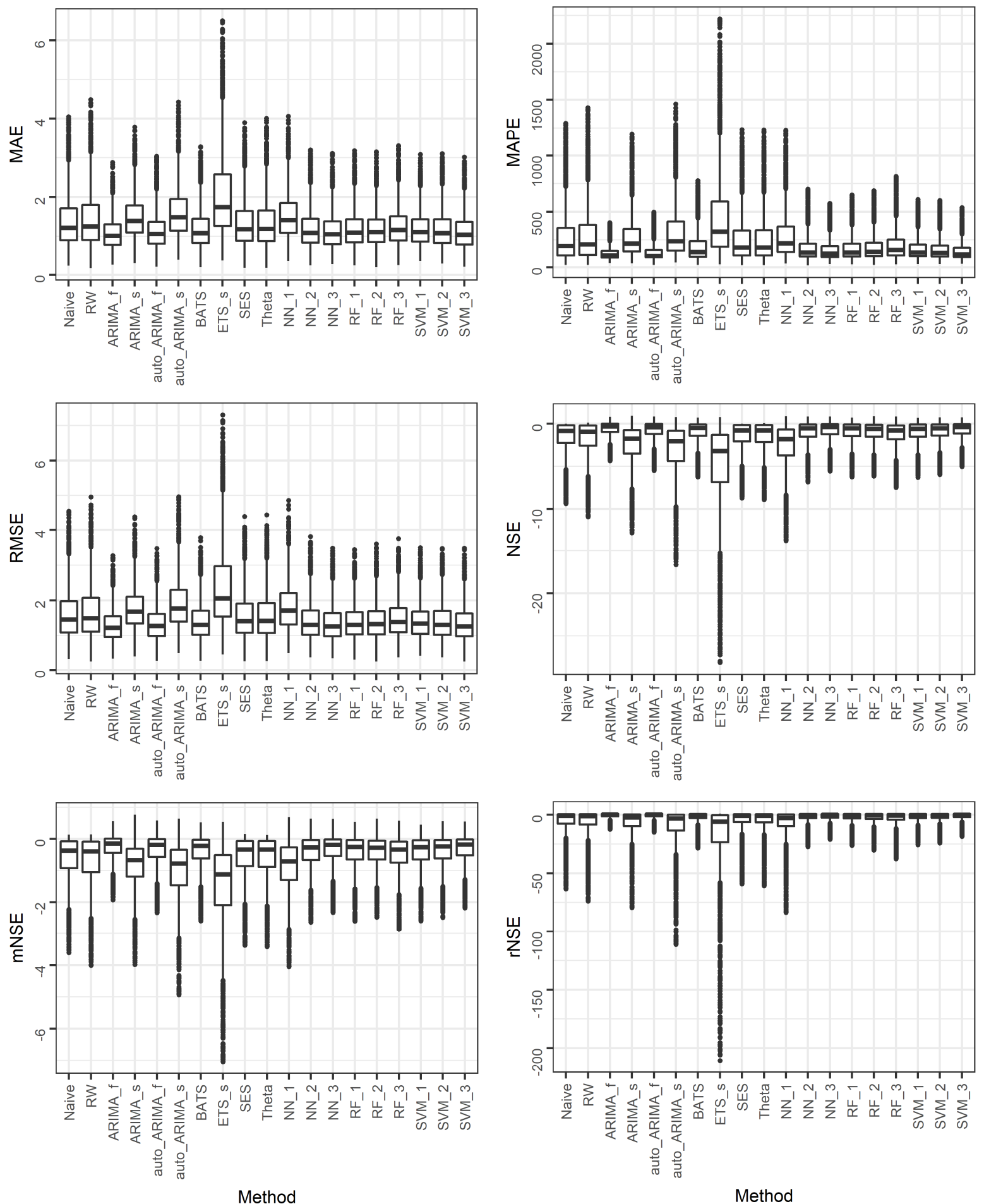

Figure 3. Side-by-side boxplots for the comparative assessment of the forecasting methods regarding their performance within the SE_1a simulation experiment (part 1). The far outliers have been removed from these side-by-side boxplots. 

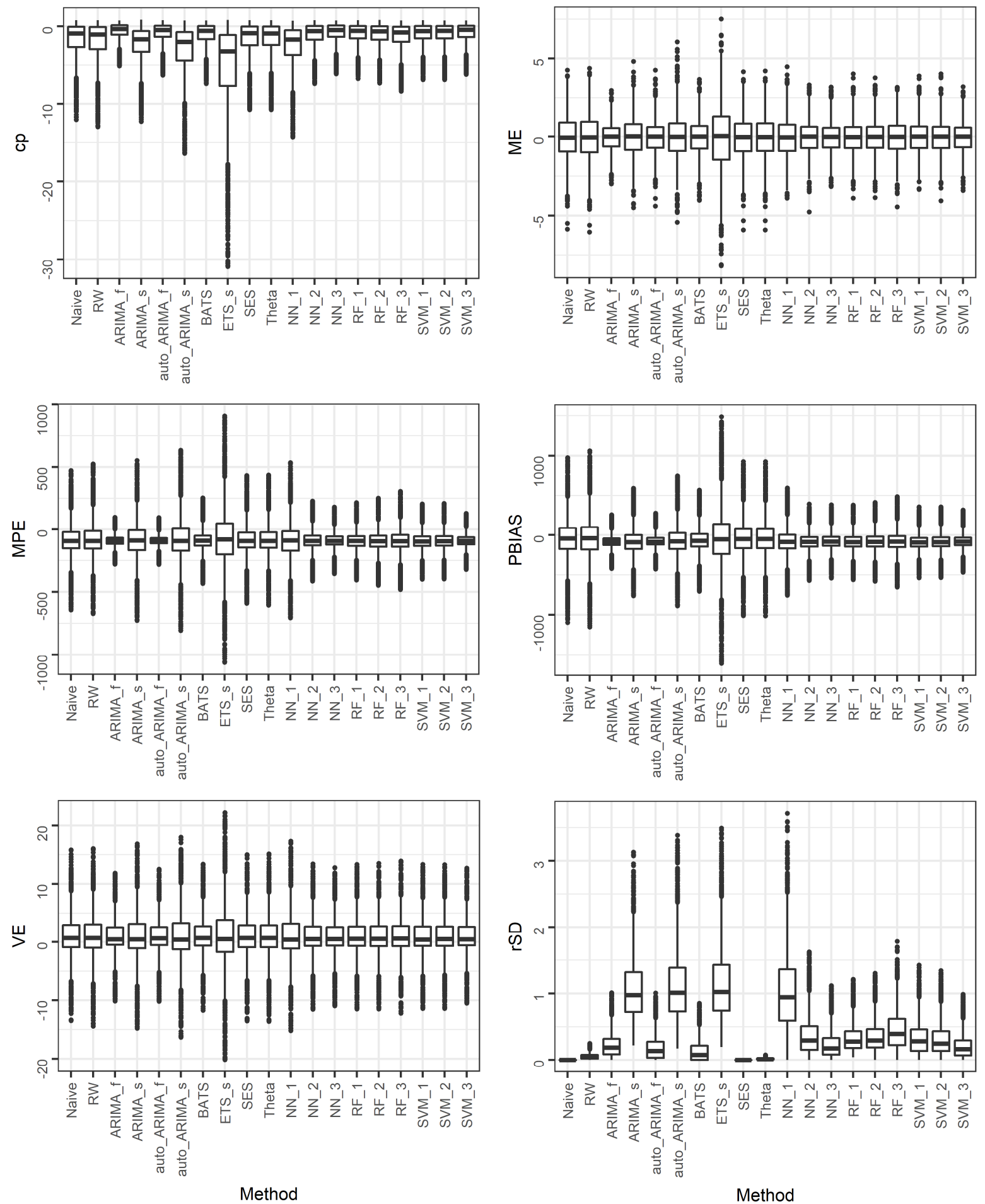

Figure 4. Side-by-side boxplots for the comparative assessment of the forecasting methods regarding their performance within the SE_1a simulation experiment (part 2). The far outliers have been removed from these side-by-side boxplots. 

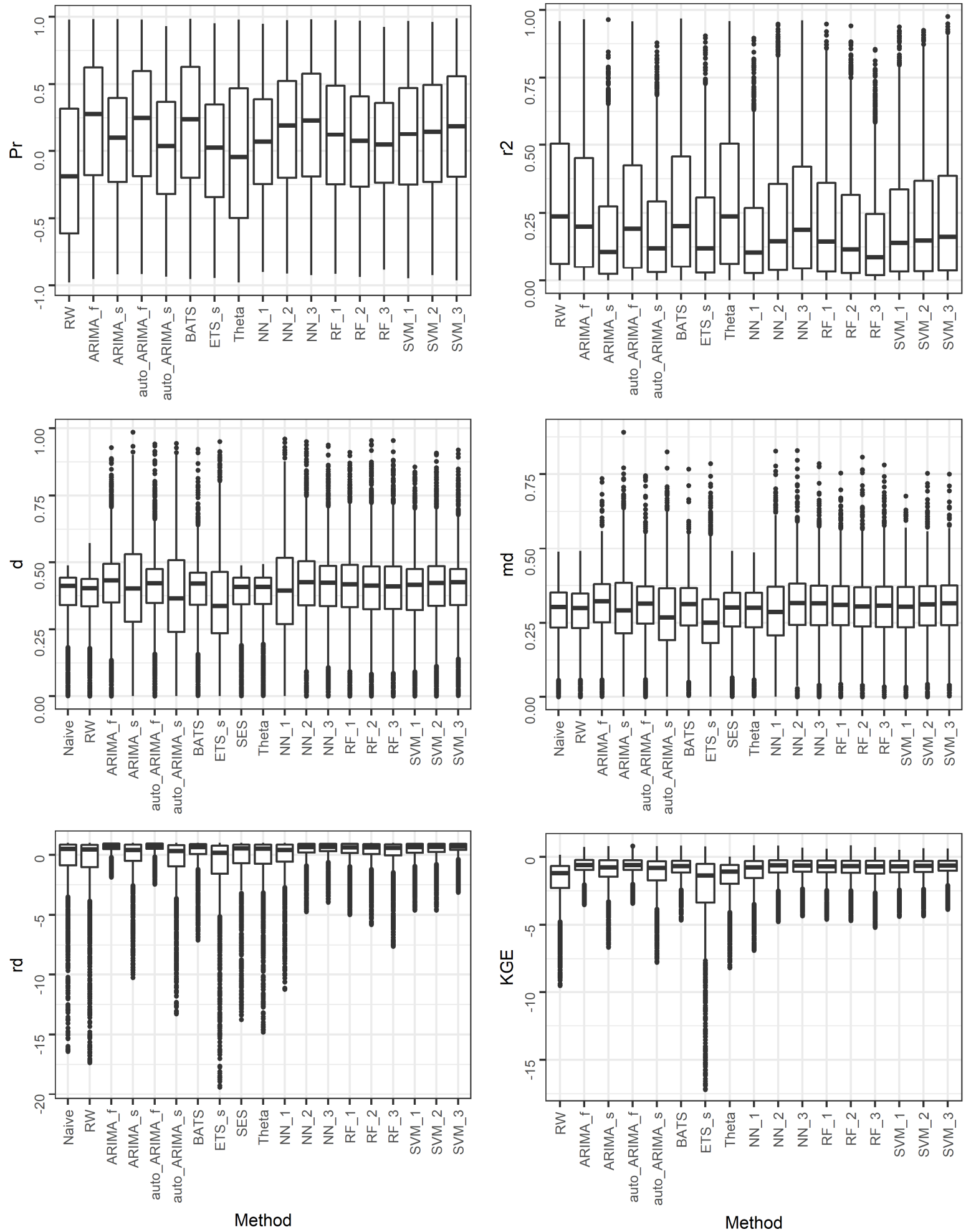

Figure 5. Side-by-side boxplots for the comparative assessment of the forecasting methods regarding their performance within the SE_1a simulation experiment (part 3). Concerning the boxplots of the rd and KGE metrics, the far outliers have been removed. The values of the Pr, r2 and KGE metrics computed for the Naïve and SES forecasting methods are infinite and, thus, excluded from the respective side-by-side boxplots. 
(a)

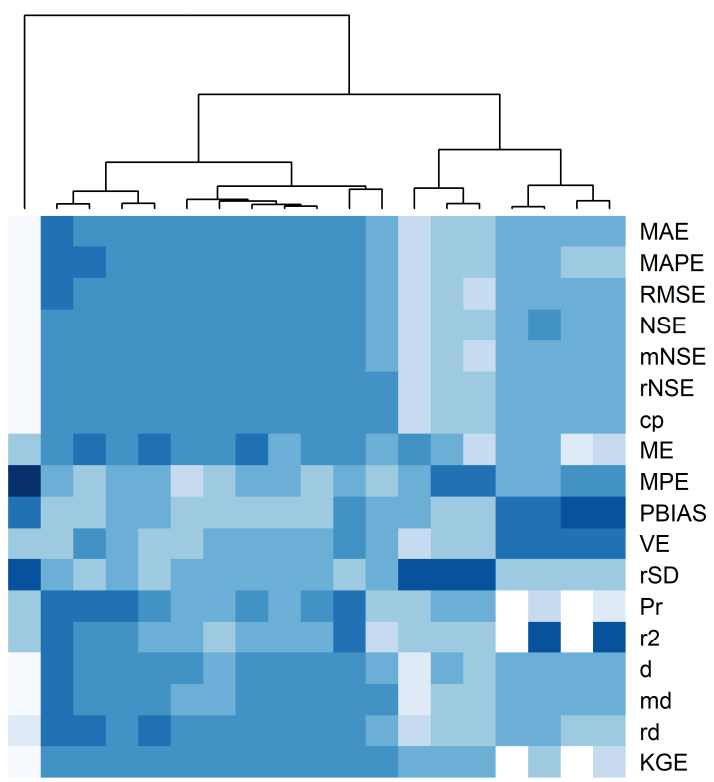

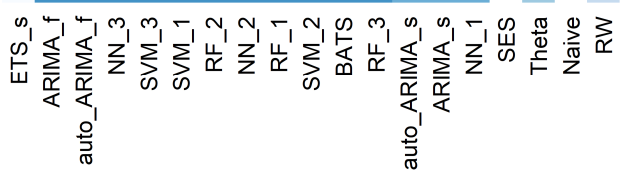

(c)

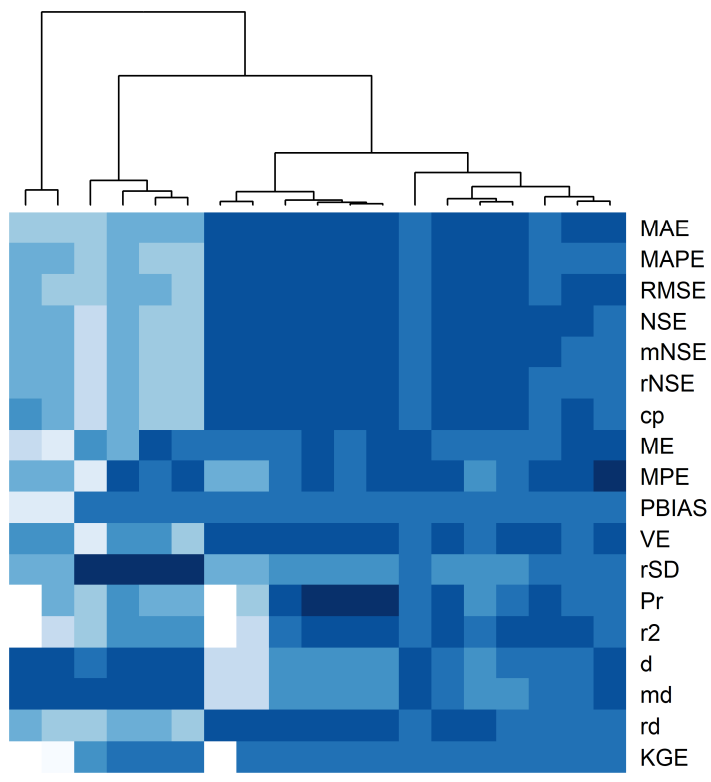

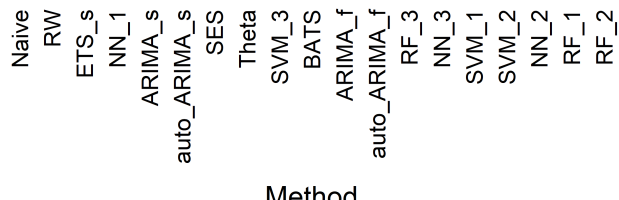

(b)

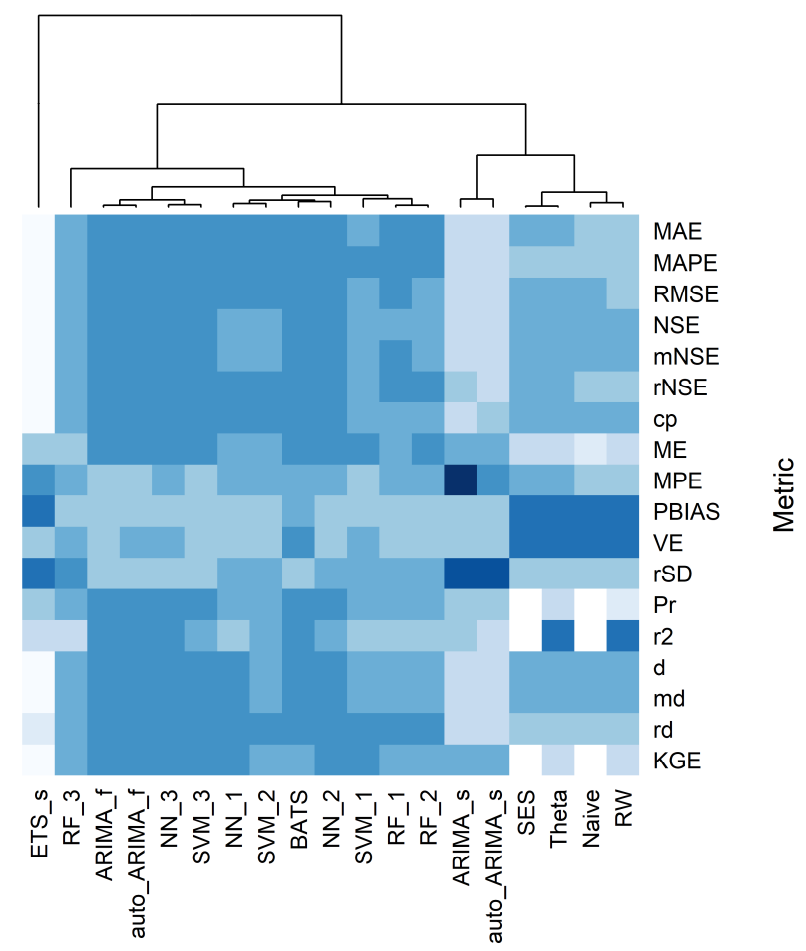

(d)

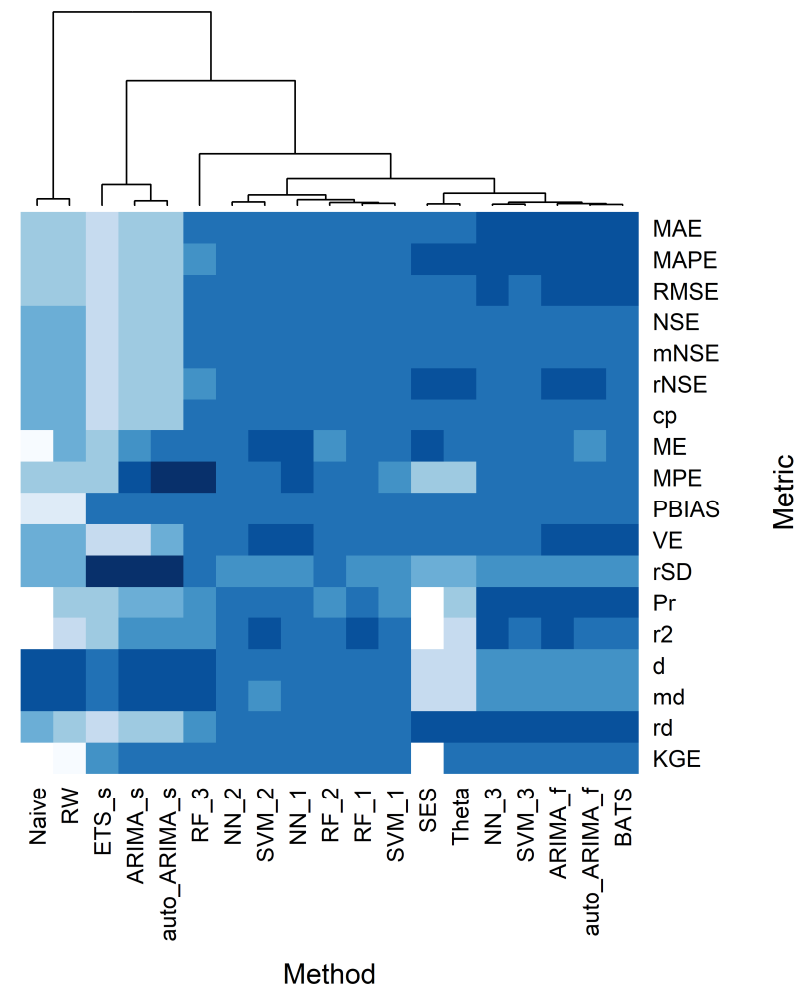

Figure 6. Heatmaps for the comparative assessment of the forecasting methods within the (a) SE_1a, (b) SE_1b, (c) SE_2a, (d) SE_2b simulation experiments according to the median values of the metrics and the conditions listed on Table 6. The infinite values of the Pr, r2 and KGE metrics for the Naïve and SES forecasting methods are not taken into consideration during the comparative assessment and are imprinted with white colour. 


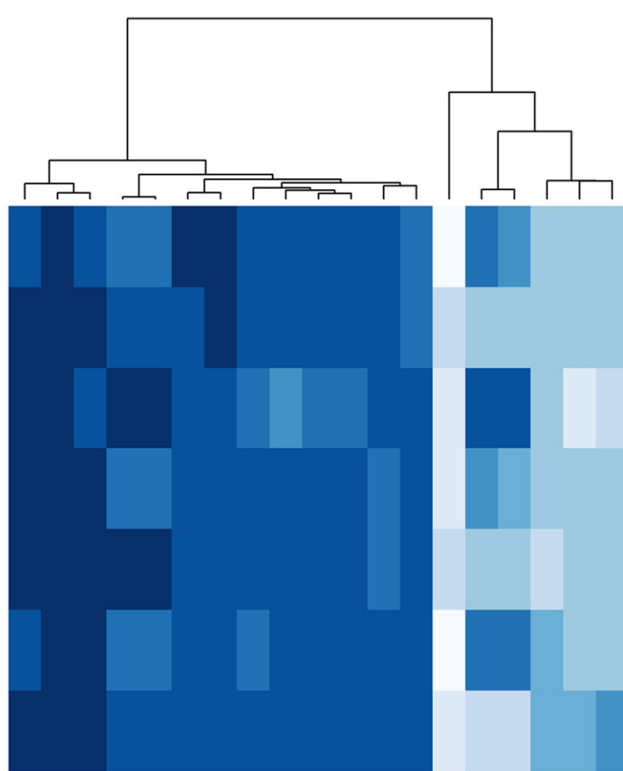

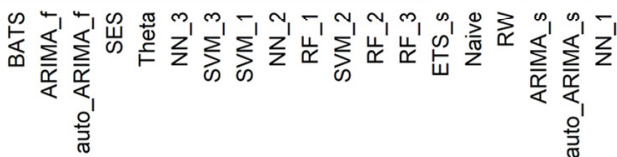

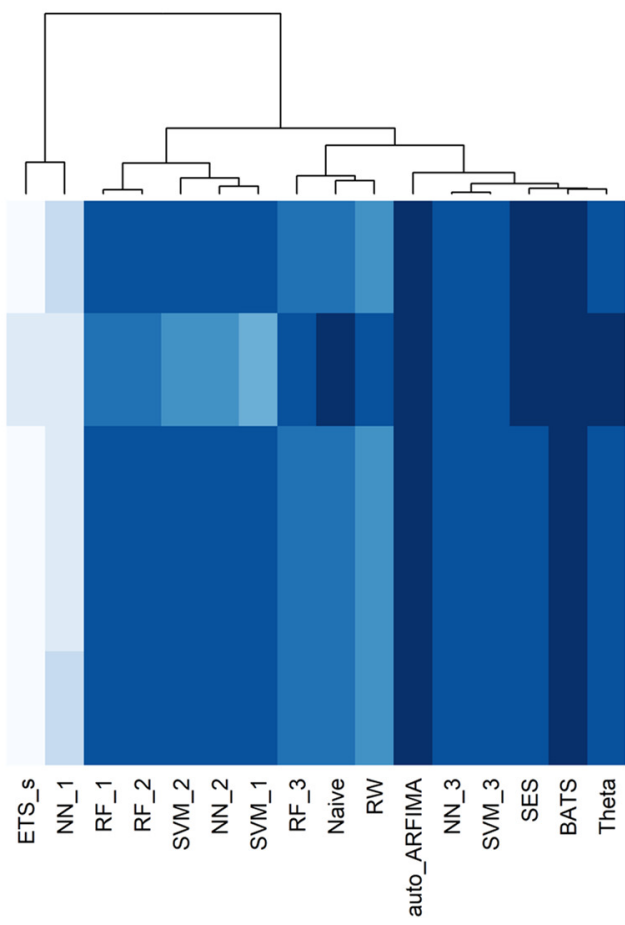

Method

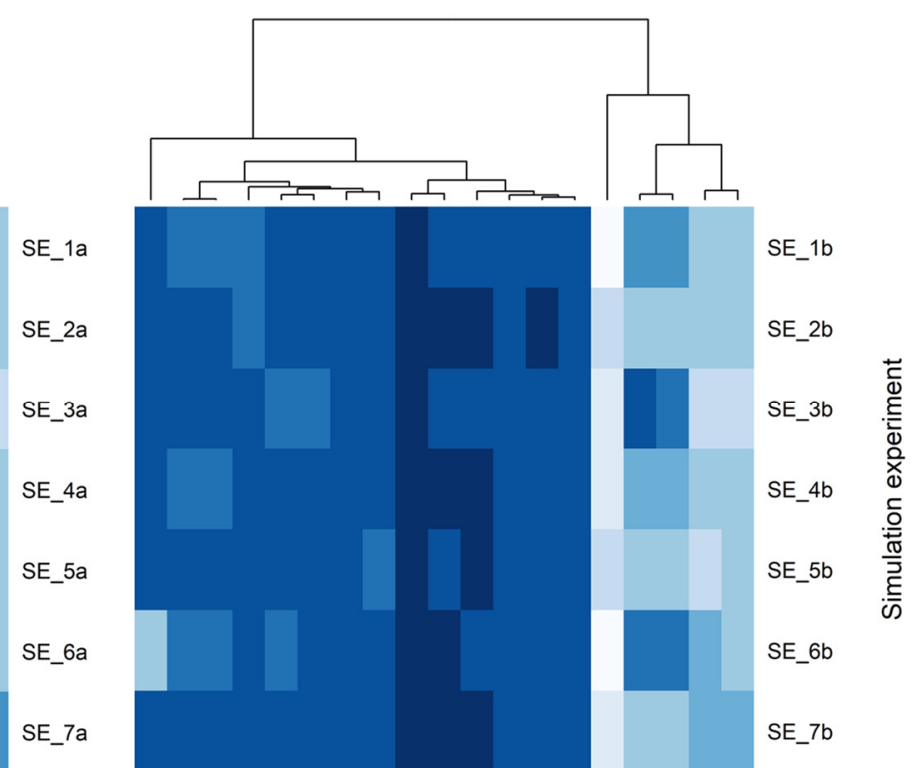

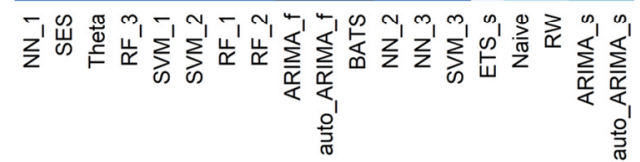

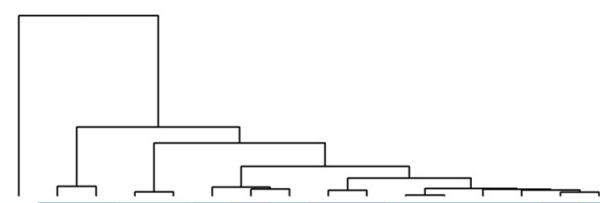

SE_8a

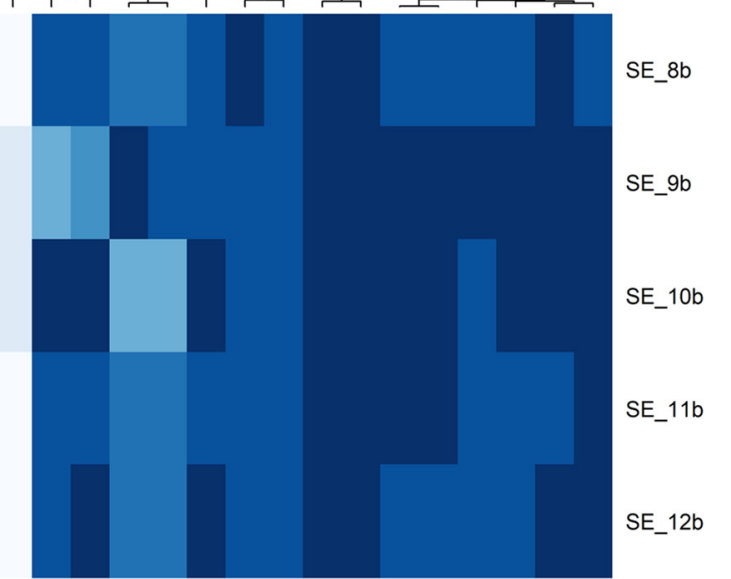

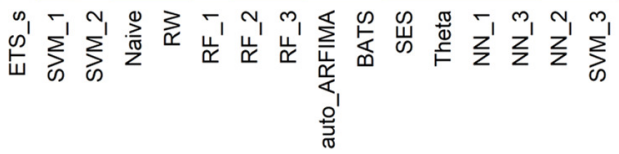

Method

Figure 7. Heatmaps for the comparative assessment of the forecasting methods according to the median values of the RMSE metric and the condition stated on Table 6. 

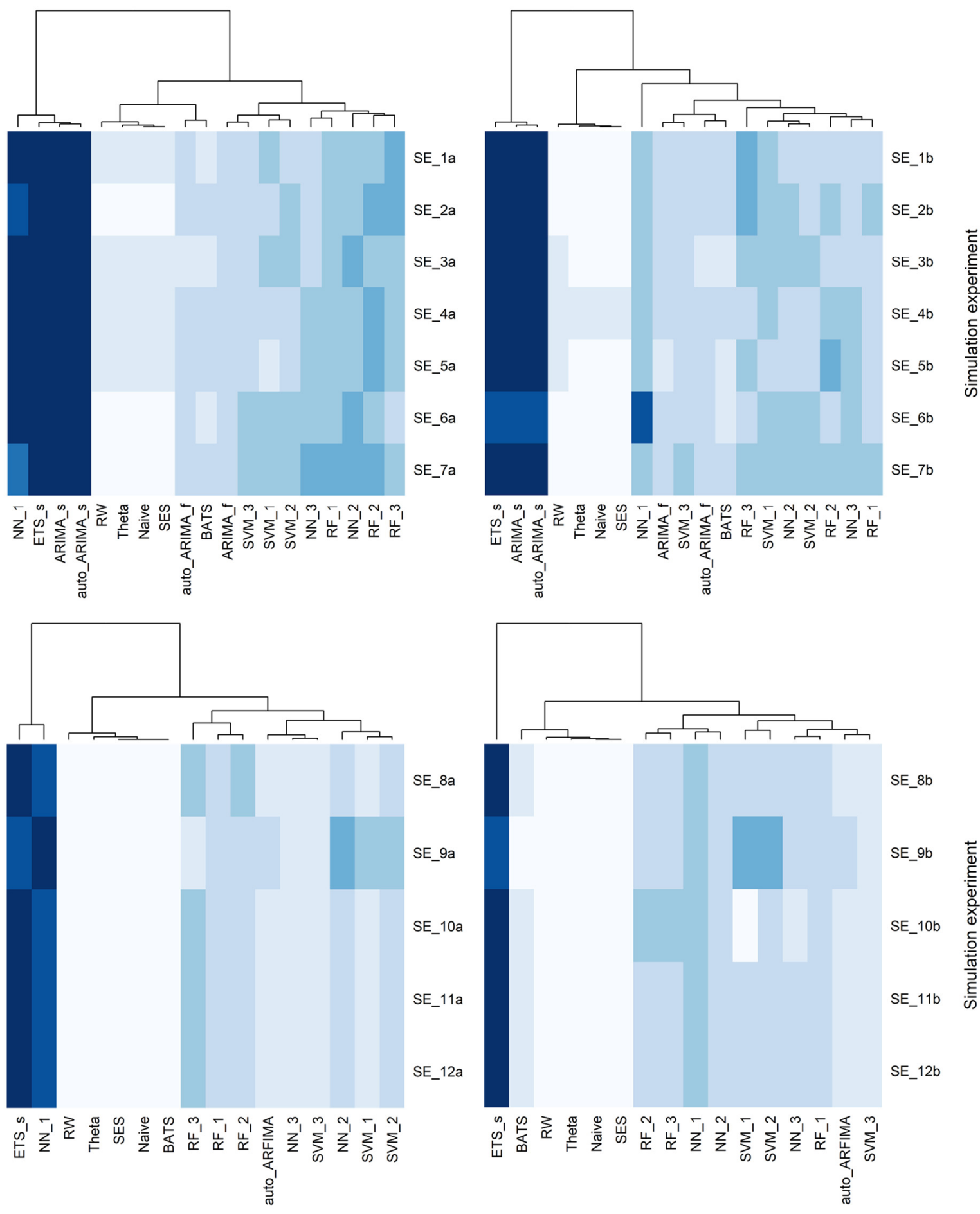

Method

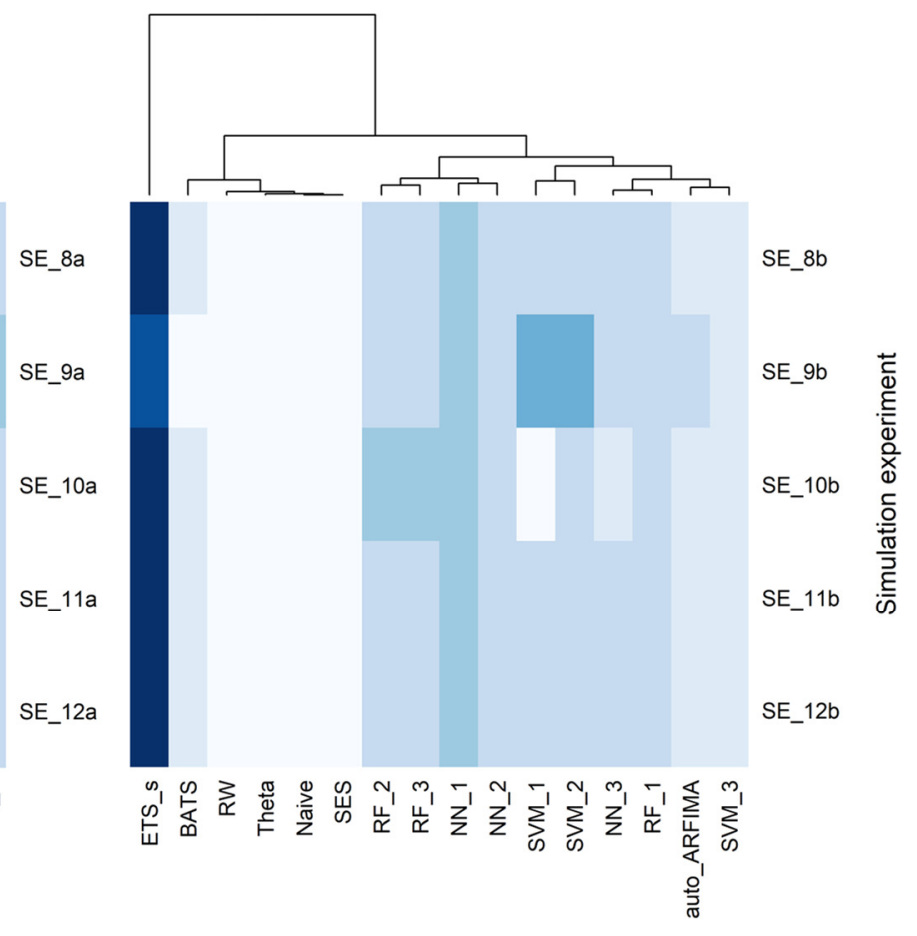

Method

Figure 8. Heatmaps for the comparative assessment of the forecasting methods according to the median values of the rSD metric and the condition stated on Table 6. 

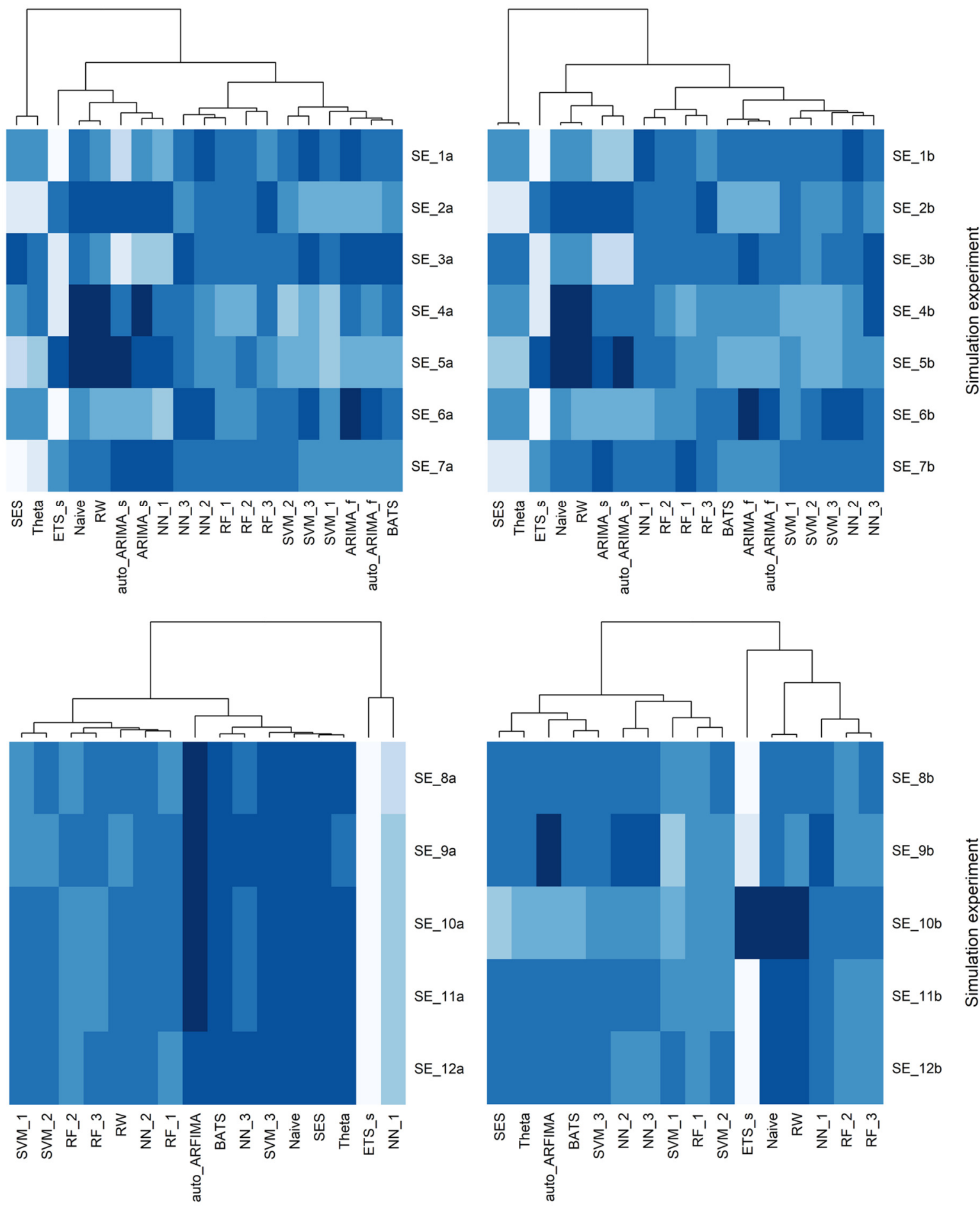

Method
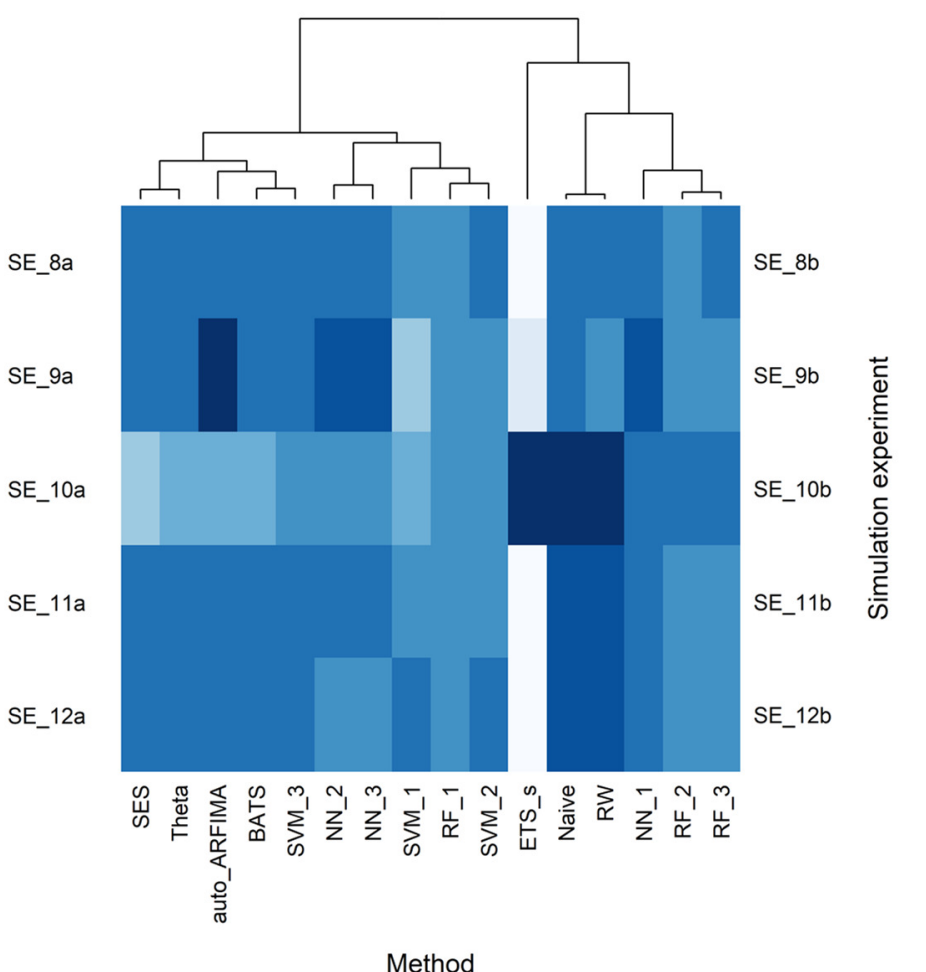

Figure 9. Heatmaps for the comparative assessment of the forecasting methods according to the median values of the d metric and the condition stated on Table 6 .

By the examination of Figure 6 (or Figures S.1-S.6) we observe that the image provided by the metrics and the resulted clustering of the forecasting methods can also vary from the one simulation experiment to the other, while by the examination of Figures 7-9 (or Figures S.7-S.24) we observe that the differences are more due to the information 
provided by specific metrics or due to specific forecasting methods. In fact, the heatmaps formed for the MAE, MAPE, RMSE, NSE, mNSE, rNSE, cp and KGE are smoother than those formed for the remaining metrics. In particular, the pictures obtained from the ME, MPE, VE, r2, d and md metrics are the most dispersed. On the other hand, the Naïve, RW, ARIMA_S, auto_ARIMA_s, ETS_S, SES, Theta and NN_1 forecasting methods are more likely to have a varying performance. For example, we observe that Naïve and RW exhibit rather the best average-case performance in terms of $d$ (see Figure 9) and md (see Figure S.22), while they have either bad, moderate or good average-case performance in terms of MAE, MAPE, PBIAS and VE depending on the simulation experiment (see Figures S.7, S.8, S.16 and S.17 respectively). The same applies to SES and Theta in terms of $d$, etc. We also note that forecasting methods resulting from the implementation of the same algorithm can exhibit a far distant or always close performance depending on the algorithm. For instance, NN_1 and NN_2 (or NN_3) may differ with each other to a great extent, a fact also applying to ARIMA_s and ARIMA_f, but not to the RF and SVM forecasting methods. Regarding NN_1, we observe that length of the time series largely affects its performance in a systematic way, while the performance of the rest forecasting methods is less or even slightly affected. The latter effect depends on the forecasting method, as well as on the simulated process. In detail, the NN_1 forecasting method exhibits a bad performance with respect to Type 1 accuracy (and a good one in terms of rSD; see Figure 8), when applied to the time series of 100 values, while its performance becomes good with respect to Type 1 accuracy (and bad in terms of rSD), when applied to the time series of 300 values. The latter observation might apply to a small extent to some of the remaining ML methods.

Finally, we summarize some important information about the best performing forecasting methods in terms of Type 1 accuracy. A good performance with respect to this criterion is a major pursuance in most of the forecasting applications. In terms of MAE (see Figure S.7) BATS is very close to the ARIMA_f, auto_ARIMA_f and auto_ARFIMA benchmarks, while SES, Theta and all the ML methods except for NN_1 have always a good or moderate performance. With respect to the MAPE metric (see Figure S.8) SVM_3 and BATS are mostly close to ARIMA_f, auto_ARIMA_f and auto_ARFIMA, and NN_2, NN_3, RF_1, RF_2, RF_3, SVM_1, SVM_2, SVM_3, SES and Theta are well performing for the greatest part of the simulation experiments. The same observations apply with respect to RMSE (see Figure 7). Nevertheless, NN_2 and NN_3 are rather very close to the good 
benchmarks as well. Regarding the NSE, mNSE, rNSE and cp values (see Figures S.10, S.11, S.12 and S.13 respectively), most of the stochastic and ML methods are competitive to each other and to the good benchmarks. The only ones that are not competitive are the simulation models, the simple benchmarks and NN_1, the latter when applied to time series of 100 values.

\subsection{Real-world experiment}

In full correspondence to the simulation experiments, the results of the real-word experiment are presented in both quantitative and qualitative forms. In Figure 10 we present the side-by-side boxplots of the MAPE, NSE, cp, MPE, $d$ and KGE values. Additionally, in Table 9 we present the median values of the dimensionless metrics, while in Figure 11 the average-case rankings of the forecasting methods. Here as well, we observe small differences between most of the methods, especially with respect to specific metrics (e.g. MAPE, cp, MPE, d). For example, the median values of MAPE computed for auto_ARFIMA, BATS, SES, Theta, NN_3, RF_1, SVM_1, SVM_2 and SVM_3 are very close to each other. The same applies to the median values of NSE computed for the same methods, although the differences in the respective side-by-side boxplots seem to be larger in the latter case than in the former. Because of the small differences in the performance of the forecasting methods, the median metric values of Table 9 (e.g. the median MAPE values) may result to a different ranking of the forecasting methods than the average-case ranking presented in Figure 11. We note that values of NSE, most of which are lower than 0 (see Table 9 and Figure 10), should not be surprising, since the examined problem differs from the traditional rainfall-runoff problem. In the latter exogenous variables are incorporated with significant increase in the available information (see also the discussion in Section 4).

Furthermore, while the average-case rankings with respect to accuracy mostly favour stochastic methods (SES, Theta, auto_ARFIMA and BATS), SVM_1 is also ranked amongst the best performing methods. In more detail, SES is ranked first according to MAE, RMSE, NSE, mNSE, cp, ME, MPE, PBIAS and VE, but it is worse than SVM_1 and SVM_2, and SVM_1, SVM_2 and SVM_3 according to MAPE and rNSE respectively. With respect to the latter metrics, the best performing method is BATS. This method has a rather moderate overall performance in terms of accuracy. The less accurate methods, on the other hand, are Naïve, RW, ETS_s and NN_1, as it is expected from the simulation experiments. With 
respect to the remaining criteria, SES is clearly the worst performing method, while Theta, Naïve, BATS, SVM_1, NN_3 and auto_ARFIMA are also ranked behind the remaining ML methods, amongst which NN_1 is mostly ranked first.
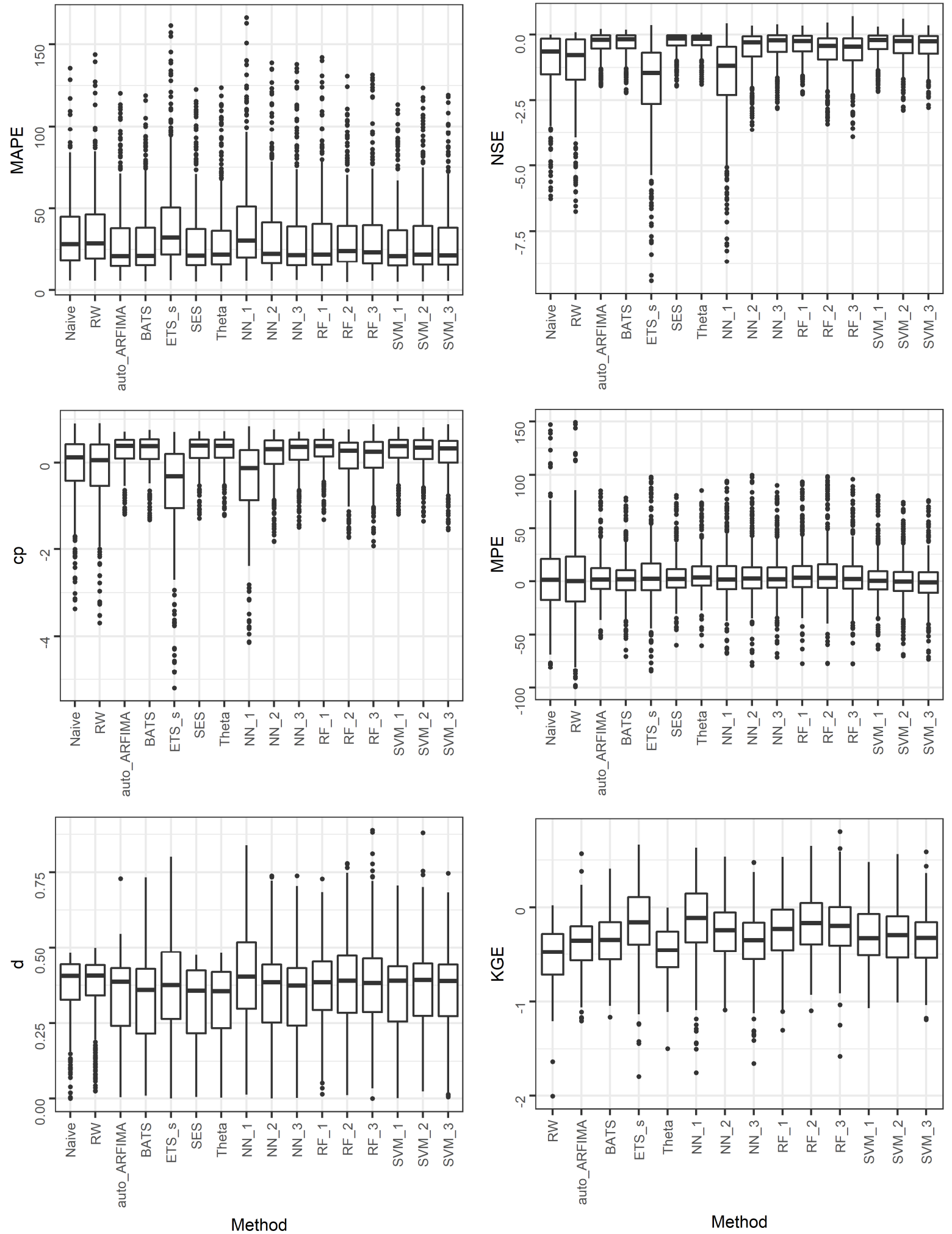

Figure 10. Side-by-side boxplots for the comparative assessment of the forecasting methods regarding their performance within the real-word experiment. The far outliers have been removed from these side-by-side boxplots. 
Table 9. Median values of the dimensionless metrics computed within the real-word experiment.

\begin{tabular}{|c|c|c|c|c|c|c|c|c|c|c|c|c|c|c|c|c|}
\hline & $\sum_{\pi}^{0}$ & $\varliminf_{a}$ & 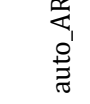 & 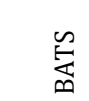 & $\begin{array}{c}\omega_{1}^{\prime} \\
\omega_{|r|}^{\prime}\end{array}$ & 苫 & $\begin{array}{l}\frac{\pi}{\mathbb{2}} \\
\stackrel{E}{E}\end{array}$ & $\begin{array}{l}r_{1}^{\prime} \\
z^{\prime}\end{array}$ & $\begin{array}{l}N_{1} \\
z^{\prime}\end{array}$ & $\begin{array}{l}m_{1} \\
z^{\prime}\end{array}$ & $\frac{\vec{r}}{\pi^{\prime}}$ & $\begin{array}{l}N_{1} \\
\frac{1}{\simeq 2}\end{array}$ & $\begin{array}{l}m_{1} \\
\simeq \widetilde{L}\end{array}$ & $\sum_{i=1}^{-1}$ & $\sum_{i=1}^{N}$ & $\sum_{i=1}^{m}$ \\
\hline MAPE & 29.21 & 29.83 & 22.04 & 22.04 & 33.54 & 22.02 & 22.83 & 32.30 & 24.05 & 22.95 & 22.80 & 25.39 & 25.17 & 22.06 & 22.46 & 22.15 \\
\hline NSE & -0.72 & -0.84 & -0.20 & -0.19 & -1.53 & -0.17 & -0.18 & -1.26 & -0.33 & -0.22 & -0.26 & -0.46 & -0.48 & -0.22 & -0.25 & -0.26 \\
\hline mNSE & -0.27 & -0.31 & -0.07 & -0.07 & -0.59 & -0.06 & -0.07 & -0.51 & -0.14 & -0.09 & -0.12 & -0.21 & -0.22 & -0.07 & -0.10 & -0.11 \\
\hline rNSE & -0.81 & -0.90 & -0.35 & -0.39 & -2.18 & -0.35 & -0.45 & -1.83 & -0.59 & -0.45 & -0.45 & -0.84 & -0.82 & -0.37 & -0.39 & -0.38 \\
\hline $\mathrm{cp}$ & 0.09 & 0.03 & 0.39 & 0.38 & -0.33 & 0.39 & 0.38 & -0.16 & 0.30 & 0.36 & 0.37 & 0.27 & 0.24 & 0.38 & 0.32 & 0.33 \\
\hline MPE & 2.83 & 1.47 & 2.99 & 2.20 & 4.54 & 3.32 & 5.07 & 2.94 & 4.61 & 3.36 & 4.34 & 3.81 & 3.61 & 2.51 & 1.25 & 0.41 \\
\hline PBIAS & -6.34 & -6.34 & -3.14 & -4.25 & -2.78 & -2.86 & -1.67 & -3.05 & -2.09 & -2.41 & -1.27 & -2.50 & -3.04 & -3.89 & -5.41 & -5.88 \\
\hline VE & 0.71 & 0.71 & 0.78 & 0.78 & 0.68 & 0.78 & 0.78 & 0.69 & 0.76 & 0.78 & 0.78 & 0.75 & 0.76 & 0.78 & 0.77 & 0.78 \\
\hline rSD & 0.00 & 0.03 & 0.05 & 0.00 & 1.00 & 0.00 & 0.01 & 0.94 & 0.21 & 0.05 & 0.24 & 0.44 & 0.41 & 0.00 & 0.13 & 0.08 \\
\hline $\operatorname{Pr}$ & $\infty$ & -0.05 & 0.06 & 0.04 & 0.00 & $\infty$ & -0.04 & 0.08 & 0.08 & 0.02 & 0.07 & 0.07 & -0.01 & 0.10 & 0.04 & 0.05 \\
\hline r2 & $\infty$ & 0.07 & 0.06 & 0.05 & 0.06 & $\infty$ & 0.07 & 0.05 & 0.06 & 0.06 & 0.06 & 0.07 & 0.06 & 0.07 & 0.07 & 0.07 \\
\hline $\mathrm{d}$ & 0.41 & 0.41 & 0.39 & 0.36 & 0.38 & 0.36 & 0.36 & 0.40 & 0.39 & 0.37 & 0.39 & 0.39 & 0.38 & 0.39 & 0.39 & 0.39 \\
\hline md & 0.31 & 0.31 & 0.28 & 0.28 & 0.29 & 0.27 & 0.26 & 0.30 & 0.29 & 0.28 & 0.28 & 0.30 & 0.30 & 0.29 & 0.30 & 0.29 \\
\hline $\mathrm{rd}$ & 0.29 & 0.30 & 0.25 & 0.26 & 0.29 & 0.22 & 0.18 & 0.33 & 0.28 & 0.22 & 0.28 & 0.29 & 0.32 & 0.29 & 0.34 & 0.36 \\
\hline KGE & $\infty$ & -0.47 & -0.35 & -0.34 & -0.16 & $\infty$ & -0.46 & -0.12 & -0.24 & -0.35 & -0.23 & -0.17 & -0.20 & -0.32 & -0.29 & -0.32 \\
\hline
\end{tabular}

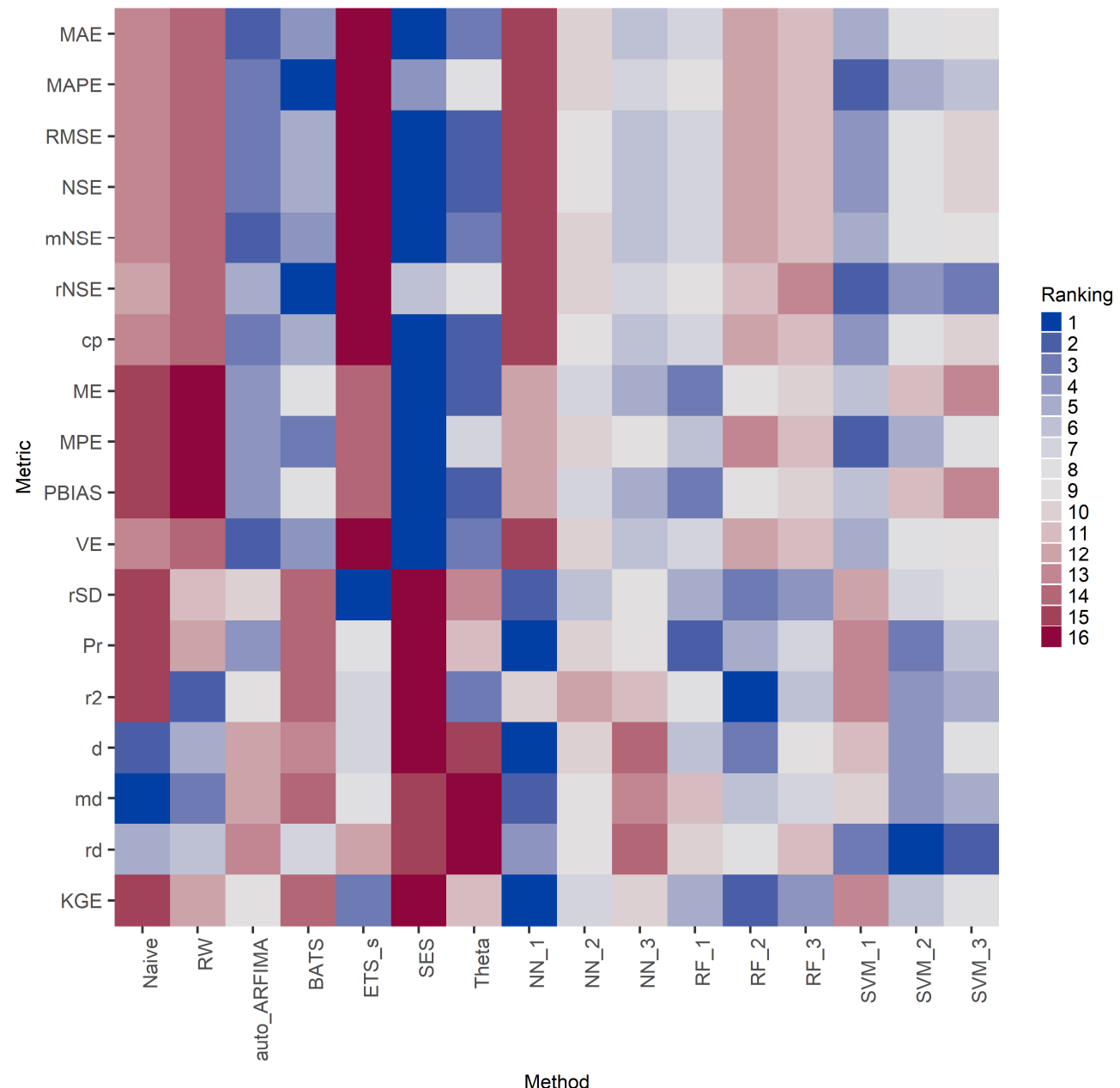

Figure 11. Heatmap for the comparative assessment of the forecasting methods within the real-world experiment according to their average-case rankings. The latter are based on the values of the metrics and the conditions listed on Table 6. The Naïve and SES forecasting methods are ranked $15^{\text {th }}$ and $16^{\text {th }}$ according to rSD, Pr, r2 and KGE. Their rSD values are 0 , while the Pr, r2 and KGE values are infinite. 


\section{Discussion}

\subsection{Contribution in hydrology and beyond}

The present study contributes by developing a detailed framework for assessing forecasting techniques in hydrology. Furthermore, its findings can provide new insights into the nature of short hydrological time series forecasting, while they concern all natural processes that could be modelled by stationary processes and all possible time scales. A first view of the results suggests that the differences in the performance of the forecasting methods are mostly small (insignificant for hydrometeorological applications), while the stochastic and ML methods can share a quite similar performance when forecasting hydrological time series of small length. In fact, methods from both these categories are found to perform better or worse mainly depending on the metric, but on the experiment as well. Regarding the Type 1 accuracy, in the simulation experiments BATS is always close to the ARIMA_f, auto_ARIMA_f and auto_ARFIMA benchmarks, probably because it uses information from an ARMA model, while most of the ML methods (e.g. NN_3 and SVM_3) are amongst the best performing and often better than SES and Theta. Nevertheless, in the real-world experiment SES is mostly ranked first, followed by auto_ARFIMA, BATS, SVM_1 and Theta, while NN_3, RF_1, SVM_2, and SVM_3 are also close to the latter methods. This outcome might mean that for a different sample of river discharge time series, the average-case rankings would differ as well, and that there might be no particular reason to choose some methods over others for this specific process. Given the claims that in linear situations (e.g. the simulation experiments of this study) the ML methods are more likely to be inferior to the stochastic ones, while in non-linear situations, as it could apply to river discharge processes, the ML methods are more likely to outperform, the algorithmically obtained results of the present study are even more interesting. Noteworthy is also the fact that our results differ from the results of Makridakis et al. (2018), which favour the stochastic methods, probably due to the different experimental settings.

In this view, we would like to emphasize that the ML algorithms are accurate enough, while a worth-mentioning particularity of theirs is perhaps related to the concomitant to the use of many lagged variables decrease of the fitting set (for more details, see Tyralis and Papacharalampous (2017)) and is largely perceivable through the examination of the results obtained for NN_1. In detail, for the simulation experiments using time series of 
100 values, NN_1 exhibits a bad performance in terms of Type 1 accuracy (a fact not applying to NN_2 and NN_3, which use less and very few lagged variables respectively). On the contrary, for the simulation experiments using time series of 300 values, this method is amongst the most accurate ones. The same number of lagged variables is used by RF_1 and SVM_1. Nevertheless, the performance of the RF and SVM algorithms seems to be less affected by the length of the fitting set. In fact, (lagged) variable selection may significantly affect the performance of a ML algorithm (see, for example, the regression case study of Anctil et al. (2009)); therefore, it is the objective of many studies (e.g. Kohavi and John GH (1997), Tyralis and Papacharalampous (2017)).

While there are forecasting methods regularly better or worse than others with respect to specific criteria, this does not apply to all the forecasting methods neither to all the criteria. For example, we observe that Theta can exhibit good, moderate or bad averagecase performance in terms of specific metrics depending on the simulation experiment. Furthermore, sophisticated forecasting methods (such us the above mentioned ones) do not necessarily (but mostly) provide better forecasts than the simple Naïve and RW, as also shown in previous studies (e.g. Makridakis and Hibon (2000), Cheng et al. (2017)). These two methods perform almost identically in the experiments of the present study, but not for longer forecast horizons (see Papacharalampous et al. (2017b; 2018c)). Another pair of similarly performing forecasting methods is SES and Theta, as proved in Hyndman and Billah (2003).

In general, we cannot decide on a universally best or worst forecasting method (stochastic or ML), neither we can rank the forecasting methods based on the results of the simulation experiments. Even the relative metrics, i.e. the corresponding to the same criterion (see Table 6), provide measurements which lead us to different aspects of the same information to an extent larger or smaller depending on the pair of metrics considered. Some of these 18 different aspects are also conflicting to each other. Any ranking of the forecasting methods would require the a priori selection of an experiment and a criterion of interest, as well as the application of a simplification procedure (e.g. use of the median values of the selected metric) and, thus, would not be general. However, the classification of the forecasting methods is possible, though only to some extent. This classification could be based on the similar or contrasting performance of the forecasting methods with respect to the various metrics. For example, the simulation models (ARIMA_s, auto_ARIMA_s and ETS_s) exhibit the best average-case performance with 
respect to the capture of the variance, while they are clearly the worst performing in terms of Type 1 accuracy. This happens, since these two criteria are contradictory. For instance, the optimum forecast for an ARFIMA model is obtained when the innovations are set to be zero.

Our contribution in the field of hydrology also includes the implementation of several forecasting models barely used in hydrometeorological concepts, but commonly used in the forecasting field (RW, BATS, ETS, SES and Theta) or for regression purposes (RF). This innovation holds, especially if we could exclude from the hydrological literature the largescale companions of this study, i.e. Papacharalampous et al. (2017b; 2018b,c) and Tyralis and Papacharalampous (2017), while its practical value is indisputable. One could claim that there may be an undiscovered forecasting method (stochastic or ML), which will be better than the existing ones. As regards the "myth of the best method" the reader is referred to Hong and Fan (2016), who mention that the number of original techniques is countable and has been exhausted, while the hybrid techniques, i.e. combinations of original techniques, cannot improve further the forecasting performance.

Another important contribution of the present study is related to the so-called "no free lunch theorem" (Wolpert 1996). According to Wolpert (1996), in the space of all possible problem instances, there is not a model, which will always perform better than the other models, in the absence of significant information for the problem at hand. The present empirical study shows that even in the finite space of simple (simulated) and real-world time series examined here there is not an optimal forecasting solution. Finding the best algorithm mostly depends on our knowledge of the system. For example, using ARFIMA models for forecasting the ARFIMA simulated time series is obviously the best choice, due to the prior known information about the system. The other methods are equivalent in performance since they cannot incorporate this knowledge. In the specific class of hydrological processes forecasting finding information about the examined system could be possible, e.g. with the application of principles of physics, such as the maximum entropy principle, incorporation of information from deterministic models (e.g. Tyralis and Koutsoyiannis 2017), understanding the processes from a chaotic perspective (Sivakumar 2004) and other approaches. Obviously, the knowledge of the system is not simply equivalent to the knowledge of its statistical properties, e.g. the mean, variance, the autocorrelation function etc., but should be deeper. Therefore, the frequently met in 
the literature of the hydrological science blind use of forecasting methods is not suggested.

Additionally, it seems that major advancements in the time series forecasting performance of all methods can be achieved by incorporating appropriate exogenous variables in the model, while the potential for improving their performance in univariate time series forecasting seems limited. The latter in our opinion is also due to the nature of the problem, which is simple. Therefore, methods that are more complicated will not necessarily yield better results. A similar example is for instance the difference in the games of tic-tac-toe and Go. The former game is simple and can be solved by simple algorithms, therefore the choice of the method is not of relevance. On the other hand, the best performance on the more complex game of Go was achieved by the use of complicated machine learning algorithms (e.g. Silver et al. (2016)).

Regarding the extent to which the conclusions could be generalizable for the forecasting of short hydrological time series, we note that the stationarity assumption and the reasoning concerning its appropriateness in the modelling of geophysical properties in Koutsoyiannis and Montanari (2015) is consistent with the no free lunch theorem. In particular, if we cannot explain the behaviour of a geophysical process based on a deterministic mechanism, then the most appropriate models are stationary. Even in cases of deterministic systems, stochastic approaches are appropriate (Koutsoyiannis 2010). This is a frequently met case in modelling of geophysical processes (i.e. there is not an adequate explanation for the behaviour of the geophysical process), proving that our conclusions could be generalizable. Finally, in practical terms the contribution of this study can be summarized as follows. A forecasting problem should be approached with more than one algorithmic solutions, i.e. using many forecasting methods (stochastic and ML), while the final judgment should always be provided by an expert.

\subsection{On the methodological approach}

The above section highlights the efficiency of our methodological approach in producing large-scale and representative for the field of hydrology results. Moreover, the real-world experiment particularly accounts for the case of river discharge forecasting. Someone who examines both the results of the simulation experiments and the real-world experiment has a more complete picture of the underlying phenomena than whom considering only the results of the simulation experiments. On the other hand, the use of 
simulated processes combined with benchmarking has proved pivotal in achieving our aim under the stationarity assumption. Additionally, the use of an adequate number of forecasting methods and metrics in the present study is also of crucial importance. Using fewer forecasting methods and fewer metrics would have led to a very different overall picture, particularly if those fewer metrics corresponded to fewer criteria. Besides, the comparison is rather the only available research method for any evaluation and, consequently, the larger its scale the more generalized the derived results. For this specific reason, the novel (mainly with respect to hydrology) methodological approach of the present study is considered appropriate for the assessment of forecasting methods in hydrology. Furthermore, the qualitative form of the results facilitates their handy examination and, thus, eases the delivery of the large-scale findings. In fact, our methodology enables the assessment of the failure risk or, alternatively worded, the available opportunities for success that accompany the use of a specific forecasting method to a significant extent, while it also leads to the recognition of several advantages/disadvantages characterizing the latter. This knowledge is fundamental to the forecasters and the users of the forecasts, since a specific forecasting method can be both useful and useless, depending on the forecasting task.

\section{Conclusions}

We conduct an extensive comparison between several stochastic and machine learning methods for the multi-step ahead forecasting of hydrological processes by performing large-scale computational experiments based on simulations under the stationarity assumption. The implemented stochastic methods include simple models, models from the frequently used families of Autoregressive Moving Average and Autoregressive Fractionally Integrated Moving Average, as well as State Space and Exponential Smoothing models, while the machine learning ones are Neural Networks, Random Forests and Support Vector Machines. The aim is to provide large-scale results, while the respective comparisons in the literature are usually based on case studies. We also run a real-world experiment on the largest river discharge dataset ever used for forecasting purposes. Despite this specific focus, the results concern all natural processes that could be modelled by stationary processes and all possible time scales. The findings suggest that stochastic and machine learning methods do not differ dramatically. In fact, methods from both these categories are found to perform equally well in univariate short time series 
forecasting. This is particularly important, because it reveals that the forecast quality is subject to certain limitations. It is also consistent with the no free lunch theorem, albeit the theorem refers to an infinite space of problems instances, while here we examined a finite space of problems. The empirical investigation showed that in the given finite space, formed by simulated and annual river discharge time series, it is still satisfied. The practical conclusion drawn from this paper is that, unless there is relevant theoretical knowledge, a forecasting problem should be algorithmically approached using many forecasting methods (stochastic and machine learning), while the final judgment should be made by an expert.

Acknowledgements: Part of the Discussion section (in particular the comments on the no free lunch theorem and the use of exogenous variables) has been inspired by the "Energy Forecasting” blog (http://blog.drhongtao.com/).

Funding: This research did not receive any specific grant from funding agencies in the public, commercial, or not-for-profit sectors.

\section{Declarations of interest: none}

Author contributions: HT conceived the idea of comparing stochastic vs machine learning methods in hydrological univariate time series forecasting using large datasets. GP designed the experiments, performed the computations and wrote the manuscript under the supervision of HT during her MSc thesis. All authors have discussed the results and edited the manuscript.

\section{Appendix A Statistical software and supplementary material}

The analyses and visualizations have been performed in R Programming Language (R Core Team 2017). We have used the contributed R packages cgwtools (Witthoft 2015), devtools (Wickham and Chang 2017), EnvStats (Millard 2013), forecast (Hyndman and Khandakar 2008; Hyndman et al. 2017), fracdiff (Fraley et al. 2012), gdata (Warnes et al. 2017), ggplot2 (Wickham 2016), HKprocess (Tyralis 2016), kernlab (Karatzoglou et al. 2004), knitr (Xie 2014, 2015, 2017), nnet (Venables and Ripley 2002), randomForest (Liaw and Wiener 2002), readr (Wickham et al. 2017), rminer (Cortez 2010, 2016) and tidyr (Wickham and Henry 2017). 
The supplementary material is available in Papacharalampous et al. (2018a). We provide the fully reproducible reports together with their codes. We also provide the reports entitled "Definition of the stochastic processes", "Definition of the metrics", "Selected figures for the qualitative comparison of the forecasting methods", which we suggest to be read alongside with Sections 2.1, 2.4 and 3.1 respectively.

\section{References}

Abrahart RJ, Anctil F, Coulibaly P, Dawson CW, Mount NJ, See LM, Shamseldin AY, Solomatine DP, Toth E, Wilby RL (2012) Two decades of anarchy? Emerging themes and outstanding challenges for neural network river forecasting. Progress in Physical Geography 36(4):480-513. doi:10.1177/0309133312444943

Abudu S, Cui C, King JP, Abudukadeer K (2010) Comparison of performance of statistical models in forecasting monthly streamflow of Kizil River, China. Water Science and Engineering 3(3):269-281. doi:10.3882/j.issn.1674-2370.2010.03.003

Ahmed NK, Atiya AF, GayarAn NE, El-Shishiny H (2010) An empirical comparison of machine learning models for time series forecasting. Econometric Reviews 29(56):594-621. doi:10.1080/07474938.2010.481556

Alpaydin E (2010) Introduction to Machine Learning, second edition. MIT Press

Anctil F, Filion M, Tournebize J (2009) A neural network experiment on the simulation of daily nitrate-nitrogen and suspended sediment fluxes from a small agricultural catchment. $\quad$ Ecological 220(6):879-887. doi:10.1016/j.ecolmodel.2008.12.021

Assimakopoulos V, Nikolopoulos K (2000) The theta model: a decomposition approach to forecasting. International Journal of Forecasting 16(4):521-530. doi:10.1016/S0169-2070(00)00066-2

Atiya AF, El-Shoura SM, Shaheen SI, El-Sherif MS (1999) A comparison between neuralnetwork forecasting techniques-case study: river flow forecasting. IEEE Transactions on Neural Networks 10(2):402-409. doi:10.1109/72.750569

Ballini R, Soares S, Andrade MG (2001) Multi-step-ahead monthly streamflow forecasting by a neurofuzzy network model. IFSA World Congress and 20th NAFIPS International Conference:992-997. doi:10.1109/NAFIPS.2001.944740

Bontempi G (2013) Machine Learning Strategies for Time Series Prediction. European Business Intelligence Summer School, Hammamet, Lecture. 2013. Available online: https://pdfs.semanticscholar.org/f8ad/a97c142b0a2b1bfe20d8317ef58527ee329 a.pdf (accessed on 14 October 2017)

Box GEP, Jenkins GM (1968) Some recent advances in forecasting and control. Journal of the Royal Statistical Society. Series C (Applied Statistics) 17(2):91-109. doi: $10.2307 / 2985674$

Breiman L (2001a) Random Forests. Machine Learning 45(1):5-32. doi:10.1023/A:1010933404324

Breiman L (2001b) Statistical modeling: The two cultures (with comments and a rejoinder by the author). Statistical Science 16(3):199-231

Carlson RF, MacCormick AJA, Watts DG (1970) Application of linear random models to four annual streamflow series. Water Resources Research 6(4):1070-1078. doi:10.1029/WR006i004p01070 
Cheng CT, Xie JX, Chau KW, Layeghifard M (2008) A new indirect multi-step-ahead prediction model for a long-term hydrologic prediction. Journal of Hydrology 361(1-2):118-130. doi:10.1016/j.jhydrol.2008.07.040

Cheng KS, Lien YT, Wu YC, Su YF (2017) On the criteria of model performance evaluation for real-time flood forecasting. Stochastic Environmental Research and Risk Assessment 31(5):1123-1146. doi:10.1007/s00477-016-1322-7

Cortez P (2010) Data mining with neural networks and support vector machines using the R/rminer tool. In: Perner P (eds) Advances in Data Mining. Applications and Theoretical Aspects. Springer Berlin Heidelberg, pp 572-583. doi:10.1007/978-3642-14400-4_44

Cortez P (2016) rminer: Data Mining Classification and Regression Methods. R package version 1.4.2. https://CRAN.R-project.org/package=rminer

Cortes C, Vapnik V (1995) Support-vector networks. Machine Learning 20(3):273-297. doi:10.1007/BF00994018

Criss RE, Winston WE (2008) Do Nash values have value? Discussion and alternate proposals. Hydrological Processes 22:2723-2725. doi:10.1002/hyp.7072

De Gooijer JG, Hyndman RJ (2006) 25 years of time series forecasting. International Journal of Forecasting 22(3):443-473. doi:10.1016/j.ijforecast.2006.01.001

De Vos NJ (2013) Echo state networks as an alternative to traditional artificial neural networks in rainfall-runoff modelling. Hydrology and Earth System Sciences 17:253-267. doi:10.5194/hess-17-253-2013

Guo J, Zhou J, Qin H, Zou Q, Li Q (2011) Monthly streamflow forecasting based on improved support vector machine model. Expert Systems with Applications 38(10):1307313081. doi:10.1016/j.eswa.2011.04.114

Gupta HV, Kling H, Yilmaz KK, Martinez GF (2009) Decomposition of the mean squared error and NSE performance criteria: Implications for improving hydrological modelling. Journal of Hydrology 377(1-2):80-91. doi:10.1016/j.jhydrol.2009.08.003

Fraley C, Leisch F, Maechler M, Reisen V, Lemonte A (2012) fracdiff: Fractionally differenced ARIMA aka ARFIMA(p,d,q) models. $R$ package version 1.4-2. https://CRAN.R-project.org/package=fracdiff

[dataset] GRDC (2017) Long-term statistics and annual characteristics of GRDC timeseries data. Online provided by the Global Runoff Data Centre of WMO. Koblenz: Federal Institute of Hydrology (BfG). [Date of retrieval:2018-01-06]. http://www.bafg.de/GRDC/EN/03_dtprdcts/32_LTMM/longtermstat_node.html

He Z, Wen X, Liu H, Du J (2014) A comparative study of artificial neural network, adaptive neuro fuzzy inference system and support vector machine for forecasting river flown in the semiarid mountain region. Journal of Hydrology 509:379-386. doi:10.1016/j.jhydrol.2013.11.054

Hong T, Fan S (2016) Probabilistic electric load forecasting: A tutorial review. International Journal of Forecasting 32(3):914-938. doi:10.1016/j.ijforecast.2015.11.011

Hong WC (2008) Rainfall forecasting by technological machine learning models. Applied Mathematics and Computation 200(1):41-57. doi:10.1016/j.amc.2007.10.046

$\mathrm{Hu}$ J, Liu J, Liu Y, Gao C (2001) EMD-KNN model for annual average rainfall forecasting. Journal of Hydrologic Engineering 18(11):1450-1457. doi:10.1061/(ASCE)HE.1943-5584.0000481 
Humphrey GB, Maier HR, Wu W, Mount NJ, Dandy GC, Abrahart RJ, Dawson CW (2017) Improved validation framework and R-package for artificial neural network models. Environmental Modelling and Software 92:82-106. doi:10.1016/j.envsoft.2017.01.023

Hyndman RJ, Athanasopoulos G (2013) Forecasting: principles and practice. OTexts: Melbourne, Australia. http://otexts.org/fpp/

Hyndman RJ, O'Hara-Wild M, Bergmeir C, Razbash S, Wang E (2017) forecast: Forecasting functions for time series and linear models. R package version 8.0. https://CRAN.Rproject.org/package=forecast

Hyndman RJ, Billah B (2003) Unmasking the Theta method. International Journal of Forecasting 19(2):287-290. doi:10.1016/S0169-2070(01)00143-1

Hyndman RJ, Khandakar Y (2008) Automatic time series forecasting: the forecast package for R. Journal of Statistical Software 27(3):1-22. doi:10.18637/jss.v027.i03

Hyndman RJ, Koehler AB, Ord JK, Snyder RD (2008) Forecasting with exponential smoothing: The state space approach. Springer - Verlag Berlin Heidelberg, pp 3-7. doi:10.1007/978-3-540-71918-2

Jain SK, Das A, Srivastava DK (1999) Application of ANN for reservoir inflow prediction and operation. Journal of Water Resources 125(5):263-271. doi:10.1061/(ASCE)0733-9496(1999)125:5(263)

Karatzoglou A, Smola A, Hornik K, Zeileis A (2004) kernlab - An S4 Package for Kernel Methods in R. Journal of Statistical Software 11(9):1-20

Khan MS, Coulibaly P (2006) Application of support vector machine in lake water level prediction. Journal of Hydrologic Engineering 11(3):199-205. doi:10.1061/(ASCE)1084-0699(2006)11:3(199)

Kim TW, Valdés JB (2003) Nonlinear model for drought forecasting based on a conjunction of wavelet transforms and neural networks. Journal of Hydrologic Engineering 8(6):319-328. doi:10.1061/(ASCE)1084-0699(2003)8:6(319)

Kişi Ö (2004) River flow modeling using artificial neural networks. Journal of Hydrologic Engineering 9(1):60-63. doi:10.1061/(ASCE)1084-0699(2004)9:1(60)

Kişi Ö (2007) Streamflow forecasting using different artificial neural network algorithms. Journal of Hydrologic Engineering 12(5):532-539. doi:10.1061/(ASCE)1084$0699(2007) 12: 5(532)$

Kişi Ö, Cimen M (2011) A wavelet-support vector machine conjunction model for monthly streamflow forecasting. Journal of Hydrology 399(1-2):132-140. doi:10.1016/j.jhydrol.2010.12.041

Kişi Ö, Cimen M (2012) Precipitation forecasting by using wavelet-support vector machine conjunction model. Engineering Applications of Artificial Intelligence 25(4):783-792. doi:10.1016/j.engappai.2011.11.003

Kişi Ö, Shiri J, Nikoofar B (2012) Forecasting daily lake levels using artificial intelligence approaches. Computers \& Geosciences 41:169-180. doi:10.1016/j.cageo.2011.08.027

Kitanidis PK, Bras RL (1980) Real time forecasting with a conceptual hydrologic model: 2. Applications and results. Water Resources Research 16(6):1034-1044. doi:10.1029/WR016i006p01034

Kohavi R, John GH (1997) Wrappers for feature subset selection. Artificial intelligence 97(1-2):273-324. doi:10.1016/S0004-3702(97)00043-X

Koutsoyiannis D (2010) HESS Opinions "A random walk on water". Hydrology and Earth System Sciences 14:585-601. doi:10.5194/hess-14-585-2010 
Koutsoyiannis D (2011) Hurst-Kolmogorov Dynamics and Uncertainty. Journal of the American Water Resources Association 47(3):481-495. doi:10.1111/j.17521688.2011.00543.x

Koutsoyiannis D, Montanari A (2015) Negligent killing of scientific concepts: the stationarity case. Hydrological Sciences Journal 60(7-8):1174-1183. doi:10.1080/02626667.2014.959959

Koutsoyiannis D, Yao H, Georgakakos A (2008) Medium-range flow prediction for the Nile: a comparison of stochastic and deterministic methods. Hydrological Sciences Journal 53(1):142-164. doi:10.1623/hysj.53.1.142

Krause P, Boyle DP, Bäse F (2005) Comparison of different efficiency criteria for hydrological model assessment. Advances in Geosciences 5:89-97

Krzysztofowicz R (2001) The case for probabilistic forecasting in hydrology. Journal of Hydrology 249(1-4):2-9. doi:10.1016/S0022-1694(01)00420-6

Lambrakis N, Andreou AS, Polydoropoulos P, Georgopoulos E, Bountis T (2000) Nonlinear analysis and forecasting of a brackish karstic spring. Water Resources Research 36(4):875-884. doi:10.1029/1999WR900353

Liaw A, Wiener M (2002) Classification and Regression by randomForest. R News 2(3):18-22

Lin JY, Cheng CT, Chau KW (2006) Using support vector machines for long-term discharge prediction. Hydrological Sciences Journal 51(4):599-612. doi:10.1623/hysj.51.4.599

Liong SY, Sivapragasam C (2002) Flood stage forecasting with support vector machines. Journal of the American Water Resources Association 38(1):173-186. doi:10.1111/j.1752-1688.2002.tb01544.x

Lu K, Wang L (2011) A novel nonlinear combination model based on support vector machine for rainfall prediction. Fourth International Joint Conference on Computational Sciences and Optimization:1343-1346. doi:10.1109/CSO.2011.50

Makridakis S, Hibon M, Lusk E, Belhadjali M (1987) Confidence intervals: An empirical investigation of the series in the M-competition. International Journal of Forecasting 3(3-4):489-508. doi:10.1016/0169-2070(87)90045-8

Makridakis S, Hibon M (2000) The M3-Competition: results, conclusions and implications. International Journal of Forecasting 16(4):451-476. doi:10.1016/S01692070(00)00057-1

Makridakis S, Spiliotis E, Assimakopoulos V (2018) Statistical and Machine Learning forecasting methods: Concerns and ways forward. PLoS ONE 13(3):e0194889. doi:10.1371/journal.pone.0194889

Maier HR, Dandy GC (2000) Neural networks for the prediction and forecasting of water resources variables: a review of modelling issues and applications. Environmental Modelling and Software 15(1):101-124. doi:10.1016/S1364-8152(99)00007-9

Millard SP (2013) EnvStats: An R Package for Environmental Statistics. Springer, New York

Mishra AK, Desai VR, Singh VP (2007) Drought forecasting using a hybrid stochastic and neural network model. Journal of Hydrologic Engineering 12(6):626-638. doi:10.1061/(ASCE)1084-0699(2007)12:6(626)

Montanari A, Rosso R, Taqqu MS (1997) Fractionally differenced ARIMA models applied to hydrologic time series: Identification, estimation, and simulation. Water Resources Research 33(5):1035-1044. doi:10.1029/97WR00043 
Montanari A, Rosso R, Taqqu MS (2000) A seasonal fractional ARIMA Model applied to the Nile River monthly flows at Aswan. Water Resources Research 36(5):1249-1259. doi:10.1029/2000WR900012

Murphy AM (1993) What is a good forecast? An essay on the nature of goodness in weather forecasting. Weather and Forecasting 8:28-293. doi:10.1175/15200434(1993)008<0281:WIAGFA $>2.0 . C 0 ; 2$

Nash JE, Sutcliffe JV (1970) River flow forecasting through conceptual models part I-A discussion of principles. Journal of Hydrology 10(3):282-290. doi:10.1016/00221694(70)90255-6

Pai PF, Hong WC (2007) A recurrent support vector regression model in rainfall forecasting. Hydrological Processes 21:819-827. doi:10.1002/hyp.6323

Papacharalampous GA (2016) Theoretical and empirical comparison of stochastic and machine learning methods for hydrological processes forecasting. MSc thesis. http://www.itia.ntua.gr/en/docinfo/1670/

Papacharalampous G, Tyralis H, Koutsoyiannis D (2017a) Comparison between stochastic and machine learning methods for hydrological multi-step ahead forecasting: All forecasts are wrong!, European Geosciences Union General Assembly 2017, Vienna, Geophysical Research Abstracts, Vol. 19, EGU2017-3068-2, doi:10.13140/RG.2.2.17205.47848

Papacharalampous GA, Tyralis H, Koutsoyiannis D (2017b) Error evolution in multi-step ahead streamflow forecasting for the operation of hydropower reservoirs. Preprints. doi:10.20944/preprints201710.0129.v1

Papacharalampous GA, Tyralis H, Koutsoyiannis D (2017c) Forecasting of geophysical processes using stochastic and machine learning algorithms. European Water 59:161-168

Papacharalampous GA, Tyralis H, Koutsoyiannis D (2018a) Comparison of stochastic and machine learning methods for multi-step ahead forecasting of hydrological processes: Supplementary material. Mendeley Data, v2. doi:10.17632/fjr8244m35.2 (This doi is reserved but not active. Please use the following link: https://data.mendeley.com/datasets/fjr8244m35/draft?a=704d13a5-ec4e-41dc9a79-abdb694b4add)

Papacharalampous GA, Tyralis H, Koutsoyiannis D (2018b) One-step ahead forecasting of geophysical processes within a purely statistical framework. Geoscience Letters 5(12). doi:10.1186/s40562-018-0111-1

Papacharalampous GA, Tyralis H, Koutsoyiannis D (2018c) Predictability of monthly temperature and precipitation using automatic time series forecasting methods. Acta Geophysica. doi:10.1007/s11600-018-0120-7

Pappenberger F, Ramos MH, Cloke HL, Wetterhall F, Alfieri L, Bogner K, Mueller A, Salamon P (2015) How do I know if my forecasts are better? Using benchmarks in hydrological ensemble prediction. Journal of Hydrology 522:697-713. doi:10.1016/j.jhydrol.2015.01.024

Patel SS, Ramachandran P (2015) A comparison of machine learning techniques for modeling river flow time series: the case of upper Cauvery river basin. Water Resources Management 29(2):589-602. doi:10.1007/s11269-014-0705-0

R Core Team (2017). R: A language and environment for statistical computing. $\mathrm{R}$ Foundation for Statistical Computing, Vienna, Austria. https://www.R-project.org/ 
Raghavendra NS, Deka PC (2014) Support vector machine applications in the field of hydrology: a review. Applied Soft Computing 19:372-386. doi:10.1016/j.asoc.2014.02.002

Ramos MH, Mathevet T, Thielen J, Pappenberger F (2010) Communicating uncertainty in hydro-meteorological forecasts: mission impossible?. Meteorological Applications 17(2):223-235. doi:10.1002/met.202

Ramos MH, Van Andel SJ, Pappenberger F (2013) Do probabilistic forecasts lead to better decisions?. Hydrology and Earth System Sciences 17:2219-2232. doi:10.5194/hess17-2219-2013

Sapankevych NI, Sankar R (2009) Time series prediction using support vector machines: a survey. IEEE Computational Intelligence Magazine 4(2):24-38. doi:10.1109/MCI.2009.932254

Shabri A, Suhartono (2012) Streamflow forecasting using least-squares support vector machines. Hydrological Sciences Journal 57(7):1275-1293. doi:10.1080/02626667.2012.714468

Shi Z, Han M (2007) Support vector echo-state machine for chaotic time-series prediction. IEEE Transactions on Neural Networks 18(2):359-372. doi:10.1109/TNN.2006.885113

Shmueli G (2010) To explain or to predict?. Statistical Science 25(3):289-310. doi:10.1214/10-STS330

Silver D, Huang A, Maddison C, Guez A, Sifre L, van den Driessche G, Schrittwieser J, Antonoglou I, Panneershelvam V, Lanctot M, Dieleman S, Grewe D, Nham J, Kalchbrenner N, Sutskever I, Lillicrap T, Leach M, Kavukcuoglu K, Graepel T, Hassabis D (2016) Mastering the game of Go with deep neural networks and tree search. Nature 529:484-489. doi:10.1038/nature16961

Sivakumar B (2004) Chaos theory in geophysics: past, present and future. Chaos, Solitons and Fractals 19(2):441-462. doi:10.1016/S0960-0779(03)00055-9

Sivapragasam C, Liong SY, Pasha MFK (2001) Rainfall and runoff forecasting with SSASVM approach. Journal of Hydroinformatics 3(3):141-152

Thissen U, Van Brakel R, De Weijer AP, Melssena WJ, Buydens LMC (2003) Using support vector machines for time series prediction. Chemometrics and Intelligent Laboratory Systems 69(1-2):35-49. doi:10.1016/S0169-7439(03)00111-4

Tyralis H (2016) HKprocess: Hurst-Kolmogorov Process. R package version 0.0-2. https://CRAN.R-project.org/package=HKprocess

Tyralis H, Koutsoyiannis D (2011) Simultaneous estimation of the parameters of the Hurst-Kolmogorov stochastic process. Stochastic Environmental Research and Risk Assessment 25(1):21-33. doi:10.1007/s00477-010-0408-x

Tyralis H, Koutsoyiannis D (2014) A Bayesian statistical model for deriving the predictive distribution of hydroclimatic variables. Climate Dynamics 42(11):2867-2883. doi:10.1007/s00382-013-1804-y

Tyralis H, Koutsoyiannis D (2017) On the prediction of persistent processes using the output of deterministic models. Hydrological Sciences Journal 62(13):2083-2102. doi:10.1080/02626667.2017.1361535

Tyralis H, Papacharalampous GA (2017) Variable selection in time series forecasting using random forests. Algorithms 10(4):114. doi:10.3390/a10040114

Valipour M, Banihabib ME, Behbahani SMR (2013) Comparison of the ARMA, ARIMA, and the autoregressive artificial neural network models in forecasting the monthly inflow of Dez dam reservoir. Journal of Hydrology 476(7):433-441. doi:10.1016/j.jhydrol.2012.11.017 
Vapnik VN (1995) The nature of statistical learning theory, first edition. Springer-Verlag New York. doi:10.1007/978-1-4757-3264-1

Vapnik VN (1999) An overview of statistical learning theory. IEEE Transactions on Neural Networks 10(5):988-999. doi:10.1109/72.788640

Venables WN, Ripley BD (2002) Modern Applied Statistics with S, fourth edition. SpringerVerlag New York. doi:10.1007/978-0-387-21706-2

Wang WC, Chau KW, Cheng CT, Qiu L (2009) A comparison of performance of several artificial intelligence methods for forecasting monthly discharge time series. Journal of Hydrology 374(3-4):294-306. doi:10.1016/j.jhydrol.2009.06.019

Warnes GR, Bolker B, Gorjanc G, Grothendieck G, Korosec A, Lumley T, MacQueen D, Magnusson A, Rogers J and others (2017) gdata: Various R Programming Tools for Data Manipulation. $\mathrm{R}$ package version 2.18.0. https://CRAN.Rproject.org/package $=$ gdata

Wei WWS (2006) Time Series Analysis, Univariate and Multivariate Methods, second edition. Pearson Addison Wesley

Weijs SV, Schoups G, Van de Giesen N (2010) Why hydrological predictions should be evaluated using information theory. Hydrology and Earth System Sciences 14:25452558. doi:10.5194/hess-14-2545-2010

Wickham H (2016) ggplot2. Springer International Publishing. https://doi.org/10.1007/978-3-319-24277-4

Wickham H, Chang W (2017) devtools: Tools to Make Developing R Packages Easier. R package version 1.13.4. https://CRAN.R-project.org/package=devtools

Wickham H, Henry L (2017) tidyr: Easily Tidy Data with 'spread()' and 'gather()' Functions. R package version 0.7.2. https://CRAN.R-project.org/package=tidyr

Wickham H, Hester J, Francois R, Jylänki J, Jørgensen M (2017) readr: Read Rectangular Text Data. R package version 1.1.1. https://CRAN.R-project.org/package=readr

Witthoft C (2015) cgwtools: Miscellaneous Tools. R package version 3.0. https://CRAN.Rproject.org/package $=$ cgwtools

Wolpert DH (1996) The Lack of A Priori Distinctions Between Learning Algorithms. Neural Computation 8(7):1341-1390. doi:10.1162/neco.1996.8.7.1341

Xie Y (2014) knitr: A comprehensive tool for reproducible research in R. In: Stodden V, Leisch F, Peng RD (eds) Implementing Reproducible Computational Research. Chapman and Hall/CRC

Xie Y (2015) Dynamic Documents with R and knitr. 2nd edition. Chapman and Hall/CRC Xie Y (2017) knitr: A General-Purpose Package for Dynamic Report Generation in R. R

Yaseen ZM, Allawi MF, Yousif AA, Jaafar O, Hamzah FM, El-Shafie A (2016) Non-tuned machine learning approach for hydrological time series forecasting. Neural Computing and Applications. doi:10.1007/s00521-016-2763-0

Yapo PO, Gupta HV, Sorooshian S (1996) Automatic calibration of conceptual rainfallrunoff models: sensitivity to calibration data. Journal of Hydrology 181(1-4):23-48. doi:10.1016/0022-1694(95)02918-4

Yevjevich VM (1987) Stochastic models in hydrology. Stochastic Hydrology and Hydraulics 1(1):17-36. doi:10.1007/BF01543907

Yu X, Liong SY (2007) Forecasting of hydrologic time series with ridge regression in feature space. Journal of Hydrology 332(3-4):290-302. doi:10.1016/j.jhydrol.2006.07.003

Zambrano-Bigiarini M (2014) hydroGOF: Goodness-of-fit functions for comparison of simulated and observed hydrological time series. $\mathrm{R}$ package version 0.3-8. https://CRAN.R-project.org/package=hydroGOF 
Zhang GP (2001) An investigation of neural networks for linear time-series forecasting. Computers \& Operations Research 28(12):1183-1202. doi:10.1016/S03050548(00)00033-2

Zhang GP, Patuwo BE, Hu MY (1998) Forecasting with artificial neural networks: The state of the art. International Journal of Forecasting 14(1):35-62. doi:10.1016/S01692070(97)00044-7 